\title{
The Radon Transform on SO(3): A Fourier Slice Theorem and Numerical Inversion
}

\author{
R. Hielscher ${ }^{1}$, D. Potts ${ }^{2}$, J. Prestin ${ }^{3}$, H. Schaeben ${ }^{4}$ and M. Schmalz ${ }^{5}$
}

${ }^{1}$ Institute of Biomathematics and Biometry, GSF - National Research Center for Environment and Health, D-85764 Neuherberg, Germany

${ }^{2}$ Faculty of Mathematics, Chemnitz University of Technology, D-09107 Chemnitz, Germany

${ }^{3}$ Institute of Mathematics, University of Lübeck, D-23560 Lübeck, Germany

${ }^{4}$ Institute of Geology, Freiberg University of Mining and Technology, D-09599 Freiberg, Germany

${ }^{5}$ Department of Computer Science, ETH Zurich, 8092 Zurich, Switzerland

email: Ralf.Hielscher@gsf.de

The inversion of the one-dimensional Radon transform on the rotation group $\mathrm{SO}(3)$ is an ill posed inverse problem which applies to X-ray tomography with polycrystalline materials. This communication presents a novel approach to the numerical inversion of the one-dimensional Radon transform on $\mathrm{SO}(3)$. Based on a Fourier slice theorem the discrete inverse Radon transform of a function sampled on the product space $\mathbb{S}^{2} \times \mathbb{S}^{2}$ of two two-dimensional spheres is determined as the solution of a minimization problem, which is iteratively solved using fast Fourier techniques for $\mathbb{S}^{2}$ and $\mathrm{SO}(3)$. The favorable complexity and stability of the algorithm based on these techniques has been confirmed with numerical tests.

\section{Introduction}

The Radon transform on $\mathrm{SO}(3)$ has recently been recognized to be instrumental for the analysis of crystallographic preferred orientation as it is the cornerstone of the relationship between a crystallographic orientation density function (ODF) and its experimentally accessible pole density functions (PDF) cf. [21, 22, 6]. Thus, the major problem to determine a reasonable ODF from experimental pole intensity data requires the inversion of the Radon transform on $\mathrm{SO}(3)$. This problem has rarely been studied with mathematical rigor even so there are several ad hoc methods most of which originate in material science, cf. $[1,13,19,10]$. 


\section{Introduction}

This communication presents a novel mathematically sound approach building on advanced methods of the inversion of the Radon transform on $\mathbb{R}^{3}$, cf. [15]. The numerical inversion of the Radon transform on the rotational group $\mathrm{SO}(3)$ is an ill posed inverse problem which requires careful analysis and design of algorithms. Therefore, we formulate a Fourier slice theorem for the Radon transform on $\mathrm{SO}(3)$ which characterizes the Radon transform as a multiplication operator in Fourier space.

Based on this characterization we define a discrete inverse Radon transform in terms of discrete Fourier transforms on $\mathrm{SO}(3)$ and $\mathbb{S}^{2} \times \mathbb{S}^{2}$, respectively, and their inverses. We define the inverse discrete Fourier transform of a function sampled on $\mathbb{S}^{2} \times \mathbb{S}^{2}$ as the solution of a minimization problem. It is iteratively solved applying fast algorithms for spherical harmonics.

In particular, we present for the first time a fast algorithm for the evaluation of the discrete inverse Radon transform in $\mathrm{SO}(3)$ based on fast Fourier techniques on the two-dimensional sphere $\mathbb{S}^{2}$ and the rotational group $\mathrm{SO}(3)$. More precisely, we rely on the nonequispaced Fourier transforms on $\mathbb{S}^{2}$ and $\mathrm{SO}(3)$, cf. $[12,8]$.

The paper is organized as follows. After introducing the major special functions on the domains $\mathbb{S}^{2}$ and $\mathrm{SO}(3)$ we define in Section 2 the Radon transform on $\mathrm{SO}(3)$ as the integral operator

$$
\mathcal{R}: L^{2}(\mathrm{SO}(3)) \rightarrow L^{2}\left(\mathbb{S}^{2} \times \mathbb{S}^{2}\right), \quad(\mathcal{R} f)(\mathbf{h}, \mathbf{r})=\frac{1}{2 \pi} \int_{G(\mathbf{h}, \mathbf{r})} f(\mathbf{g}) \mathrm{dg}
$$

where $G(\mathbf{h}, \mathbf{r})$ denotes for any $\mathbf{h}, \mathbf{r} \in \mathbb{S}^{2}$ a geodesic in $\mathrm{SO}(3)$. In Theorem 2.7 we derive a representation of the Radon transform $\mathcal{R}$ in Fourier space which might be interpreted as an analogue to the classical Fourier slice theorem of the Radon transform in $\mathbb{R}^{d}$.

We proceed by defining two finite dimensional approximations of the inverse Radon transform of a function $P \in C\left(\mathbb{S}^{2} \times \mathbb{S}^{2}\right)$ given its values at a finite set of nodes $\Gamma \subset \mathbb{S}^{2} \times \mathbb{S}^{2}$. Depending on the number of sample nodes and the dimension of the approximation space we define the approximations of the inverse Radon transform either as the solution of an interpolation problem or as a the solution of an optimization problem. We end the section by proving the basic approximation Theorem 2.11 relating the approximation error in $L^{2}(\mathrm{SO}(3))$ to the approximation error in the Fourier space corresponding to $\mathbb{S}^{2} \times \mathbb{S}^{2}$.

Section 3 is devoted to the discrete theory. First we present in Theorem 3.4 a factorization of the discrete Radon transform on $\mathrm{SO}(3)$, i.e., of the operator that evaluates the Radon transform of a polynomial on $\mathrm{SO}(3)$ in a finite set of nodes $\Gamma \subset \mathbb{S}^{2} \times \mathbb{S}^{2}$. Here we are concerned with sets of nodes that are of the special form $\Gamma=\left\{\left(\mathbf{h}_{i}, \mathbf{r}_{i j}\right) \in \mathbb{S}^{2} \times \mathbb{S}^{2} \mid i=1, \ldots, N, j=1, \ldots, N_{i}\right\}$. Based on the factorization of the discrete Radon transform the Algorithms 1 and 2 implement the direct and the adjoint forward transform with numerical complexity $\mathcal{O}\left(N L^{3}+|\Gamma|\right)$, where $L \in \mathbb{N}$ is the maximum polynomial degree used for the approximation of the Radon transform (cf. Lemma 3.6). This complexity compares favorably to the numerical complexity $\mathcal{O}\left(L^{3}|\Gamma|\right)$ of the naive algorithm. In Theorem 3.5 the algorithm for the discrete Radon transform is utilized to derive an algorithm for the computation of the inverse Radon transform that has the numerical complexity $\mathcal{O}\left(N L^{3}+|\Gamma|\right)$ per iteration. A necessary condition on the structure of the set of nodes $\Gamma \subset \mathbb{S}^{2} \times \mathbb{S}^{2}$ and in particular on the number $N \in \mathbb{N}$ such that the inverse problem is well posed is given in Theorem 3.9. 
In Section 4 we check the CGNR as well as the CGNE based algorithm for various combinations of polynomial degree and sampling sizes. In all our experiments both algorithms converge if the polynomial degree is chosen sufficiently large. However, in the case that the dimension of the approximation space is almost equal to the number of sampling nodes none of both algorithms converges. A similar result for the case of approximation on the sphere can be found in [9].

In the last Section 5 we address the application of the Radon transform on $\mathrm{SO}(3)$ to texture analysis. We formulate the practical problem of recovering an orientation distribution function from diffraction measurements and perform some sample calculations using the CGNR based algorithm. Since in texture analysis one is interested in the Fourier coefficients of certain orders we analyze the approximation error for each of the harmonic subspaces separately. We observe that the maximum harmonic order up to which the Fourier coefficients can be approximated is in general significantly lower than the theoretic bound given in Theorem 3.9. Moreover, we observe that the approximation error in a fixed harmonic subspace decreases if the total polynomial degree of approximation is increased. This observation has a direct consequence for the practical determination of low order Fourier coefficients in texture analysis since it suggests to choose the total polynomial degree of approximation much higher then the order of the required Fourier coefficients.

\section{Integral Operators}

Functions on $\mathbb{S}^{2}$. For the following exposition we refer to [2]. The Legendre polynomials $\mathcal{P}_{l}:[-1,1] \rightarrow \mathbb{R}, l \in \mathbb{N}_{0}$, are the key special functions in harmonic analysis on the twodimensional sphere. They are characterized as classical orthogonal polynomials on the interval $[-1,1]$ by the properties

1. $\mathcal{P}_{l}$ is a polynomial of degree $l$,

2. $\int_{-1}^{1} \mathcal{P}_{l}(t) \mathcal{P}_{l^{\prime}}(t) \mathrm{d} t=\frac{2}{2 l+1} \delta_{l, l^{\prime}}$ for $l, l^{\prime} \in \mathbb{N}_{0}$.

By property 2 the Legendre polynomials are normed to $\mathcal{P}_{l}(1)=1, l \in \mathbb{N}_{0}$. The associated Legendre functions $\mathcal{P}_{l}^{k}:[-1,1] \rightarrow \mathbb{R}, l, k \in \mathbb{N}_{0}, k \leq l$, are defined with the derivatives of the Legendre polynomials

$$
\mathcal{P}_{l}^{k}(t)=\left(\frac{(l-k) !}{(l+k) !}\right)^{1 / 2}\left(1-t^{2}\right)^{k / 2} \frac{\mathrm{d}^{k}}{\mathrm{~d} t^{k}} \mathcal{P}_{l}(t), \quad t \in[-1,1]
$$

They satisfy for all $l \in \mathbb{N}_{0}, k=0, \ldots, l$, the three term recurrence relation

$$
\begin{array}{r}
\mathcal{P}_{l-1}^{l}(t)=0, \quad \mathcal{P}_{l}^{l}(t)=\frac{\sqrt{(2 l) !}}{2^{l} l !}\left(1-t^{2}\right)^{l / 2}, \\
\mathcal{P}_{l+1}^{k}(t)=v_{l}^{k} t \mathcal{P}_{l}^{k}(t)-w_{l}^{k} \mathcal{P}_{l-1}^{k}(t), \quad t \in[-1,1],
\end{array}
$$

where

$$
v_{l}^{k}=\frac{2 l+1}{\sqrt{(l-k+1)(l+k+1)}} \quad \text { and } \quad w_{l}^{k}=\sqrt{\frac{(l-k)(l+k)}{(l-k+1)(l+k+1)}} .
$$


Let $\boldsymbol{\xi} \in \mathbb{S}^{2}$ and let $(\theta, \rho) \in[0, \pi] \times[0,2 \pi)$ be its polar coordinates, i.e.,

$$
\boldsymbol{\xi}=(\cos \rho \sin \theta, \sin \rho \sin \theta, \cos \theta)^{\mathrm{T}} .
$$

Then the spherical harmonics given as

$$
\mathcal{Y}_{l}^{k}(\boldsymbol{\xi})=\sqrt{\frac{2 l+1}{4 \pi}} \mathcal{P}_{l}^{|k|}(\cos \theta) \mathrm{e}^{\mathrm{i} k \rho}, \quad k=-l, \ldots, l,
$$

span the harmonic space $\operatorname{Harm}_{l}\left(\mathbb{S}^{2}\right)=\operatorname{span}\left\{\mathcal{Y}_{l}^{-l}, \ldots, \mathcal{Y}_{l}^{l}\right\}$ of all spherical harmonics with a fixed degree $l \in \mathbb{N}_{0}$. Moreover, the spherical harmonics (2.2) satisfy the orthogonality relationship

$$
\int_{\mathbb{S}^{2}} \mathcal{Y}_{l}^{k}(\boldsymbol{\xi}) \overline{\mathcal{Y}_{l^{\prime}}^{k^{\prime}}(\boldsymbol{\xi})} \mathrm{d} \boldsymbol{\xi}=\delta_{l l^{\prime}} \delta_{k, k^{\prime}}
$$

and the function system $\mathcal{Y}_{l}^{k}, l \in \mathbb{N}_{0}, k=-l, \ldots, l$, forms an orthonormal basis of $L^{2}\left(\mathbb{S}^{2}\right)$. The harmonic spaces $\operatorname{Harm}_{l}\left(\mathbb{S}^{2}\right), l \in \mathbb{N}_{0}$, provide a complete system of rotational invariant, irreducible subspaces of $L^{2}\left(\mathbb{S}^{2}\right)$, i.e.,

$$
L^{2}\left(\mathbb{S}^{2}\right)=\operatorname{clos}_{L^{2}} \bigoplus_{l=0}^{\infty} \operatorname{Harm}_{l}\left(\mathbb{S}^{2}\right) .
$$

Let $L \in \mathbb{N}_{0}$. Then any function

$$
f \in \bigoplus_{l=0}^{L} \operatorname{Harm}_{l}\left(\mathbb{S}^{2}\right)
$$

is called spherical polynomial of degree $L$.

For a given function $f \in L^{2}\left(\mathbb{S}^{2}\right)$ we define its Fourier sequence $\hat{f} \in \ell^{2}(I)$,

$$
I=\left\{(l, k) \in \mathbb{Z}^{2} \mid l \in \mathbb{N}_{0}, k=-l, \ldots, l\right\},
$$

as the sequence of coefficients with respect to the basis $\mathcal{Y}_{l}^{k},(l, k) \in I$, i.e.,

$$
\hat{f}(l, k)=\int_{\mathbb{S}^{2}} f(\boldsymbol{\xi}) \overline{\mathcal{Y}_{l}^{k}(\boldsymbol{\xi})} \mathrm{d} \boldsymbol{\xi}, \quad(l, k) \in I .
$$

Moreover, we define the index set

$$
I_{L}=\left\{(l, k) \in \mathbb{Z}^{2} \mid l=0, \ldots, L, k=-l, \ldots, l\right\},
$$

of the Fourier coefficients of the space of spherical polynomials of degree $L \in \mathbb{N}_{0}$ which has the dimension

$$
\left|I_{L}\right|=(L+1)^{2}
$$

Definition 2.1. We define the continuous Fourier transform $\mathcal{F}_{\mathbb{S}^{2}}$ in $L^{2}\left(\mathbb{S}^{2}\right)$ as the operator

$$
\mathcal{F}_{\mathbb{S}^{2}}: \ell^{2}(I) \rightarrow L^{2}\left(\mathbb{S}^{2}\right), \quad \hat{f} \mapsto \sum_{(l, k) \in I} \hat{f}(l, k) \mathcal{Y}_{l}^{k}
$$

By Parseval's theorem the operators $\mathcal{F}_{\mathbb{S}^{2}}, \mathcal{F}_{\mathbb{S}^{2}}^{-1}$ are well defined isometries between $L^{2}\left(\mathbb{S}^{2}\right)$ and $\ell^{2}(I)$ and we have for any function $f \in L^{2}\left(\mathbb{S}^{2}\right)$,

$$
\mathcal{F}_{\mathbb{S}^{2}}^{-1} f=\hat{f}
$$




\section{Integral Operators}

Functions on $\mathbf{S O}(3)$. By $\mathrm{SO}(3)$ we denote the Lie group of all orthogonal $3 \times 3$ matrices with determinant one. Setting

$$
J=\left\{\left(l, k, k^{\prime}\right) \in \mathbb{Z}^{3} \mid l \in \mathbb{N}_{0}, k, k^{\prime}=-l, \ldots, l\right\},
$$

we consider a basis system $D_{l}^{k k^{\prime}} \in L^{2}(\mathrm{SO}(3)),\left(l, k, k^{\prime}\right) \in J$ of harmonic functions on $\mathrm{SO}(3)$ following [23]. These functions $D_{l}^{k k^{\prime}},\left(l, k, k^{\prime}\right) \in J$ are called Wigner-D functions and are defined by the representation properties

$$
D_{l}^{k k^{\prime}}(\mathbf{g})=\int_{\mathbb{S}^{2}} \mathcal{Y}_{l}^{k^{\prime}}\left(\mathbf{g}^{-1} \boldsymbol{\xi}\right) \overline{\mathcal{Y}_{l}^{k}(\boldsymbol{\xi})} \mathrm{d} \boldsymbol{\xi}, \quad\left(l, k, k^{\prime}\right) \in J, \mathbf{g} \in \mathrm{SO}(3)
$$

The Wigner-D functions form an orthogonal basis in $L^{2}(\mathrm{SO}(3))$ with respect to the Haar measure. In particular, every function $f \in L^{2}(\mathrm{SO}(3))$ has a unique series expansion in terms of Wigner-D functions

$$
f=\sum_{\left(l, k, k^{\prime}\right) \in J} \frac{\left(l+\frac{1}{2}\right)^{\frac{1}{2}}}{2 \pi} \hat{f}\left(l, k, k^{\prime}\right) D_{l}^{k k^{\prime}}
$$

with Fourier coefficients $\hat{f}\left(l, k, k^{\prime}\right),\left(l, k, k^{\prime}\right) \in J$, given by the integrals

$$
\hat{f}\left(l, k, k^{\prime}\right)=\frac{\left(l+\frac{1}{2}\right)^{\frac{1}{2}}}{2 \pi} \int_{\mathrm{SO}(3)} f(\mathbf{g}) \overline{D_{l}^{k k^{\prime}}(\mathbf{g})} \mathrm{dg} .
$$

Note that the Wigner-D functions $D_{l}^{k k^{\prime}}$ are not normalized in the $L^{2}$-sense but satisfy

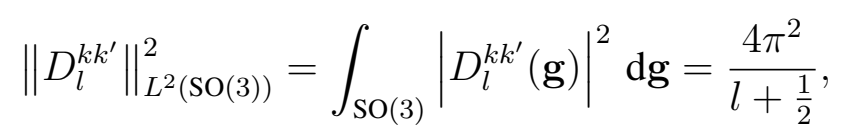

with the measure on $\mathrm{SO}(3)$ normalized to $\int_{\mathrm{SO}(3)} \mathrm{dg}=8 \pi^{2}$.

Let $l \in \mathbb{N}_{0}$. Then the harmonic space $\operatorname{Harm}_{l}(\mathrm{SO}(3))$ of degree $l$ is defined as

$$
\operatorname{Harm}_{l}(\operatorname{SO}(3))=\operatorname{span}\left\{D_{l}^{k k^{\prime}} \mid k, k^{\prime}=-l, \ldots, l\right\} .
$$

For reasons of analogy we call any function $f \in \bigoplus_{l=0}^{L} \operatorname{Harm}_{l}(\mathrm{SO}(3))$ a polynomial on $\mathrm{SO}(3)$ of degree $L \in \mathbb{N}_{0}$ and correspondingly define the truncated index set

$$
J_{L}=\left\{\left(l, k, k^{\prime}\right) \in \mathbb{Z}^{3} \mid l=0, \ldots, L, k, k^{\prime}=-l, \ldots, l\right\} .
$$

The dimension of the space of these polynomials is given by

$$
\left|J_{L}\right|=\frac{1}{3}(L+1)(2 L+1)(2 L+3)
$$

Similar to the spherical case we introduce the Fourier transform in $L^{2}(\mathrm{SO}(3))$. 
Definition 2.2. The continuous Fourier transform in $L^{2}(\mathrm{SO}(3))$ is defined as the operator

$$
\mathcal{F}_{\mathrm{SO}(3)}: \ell^{2}(J) \rightarrow L^{2}(\mathrm{SO}(3)), \quad \hat{f} \mapsto \sum_{\left(l, k, k^{\prime}\right) \in J} \frac{\left(l+\frac{1}{2}\right)^{\frac{1}{2}}}{2 \pi} \hat{f}\left(l, k, k^{\prime}\right) D_{l}^{k k^{\prime}} .
$$

By Parseval's theorem the operators $\mathcal{F}_{\mathrm{SO}(3)}, \mathcal{F}_{\mathrm{SO}(3)}^{-1}$ are well defined isometries between $L^{2}(\mathrm{SO}(3))$ and $\ell^{2}(J)$ and we have for any function $f \in L^{2}(\mathrm{SO}(3))$

$$
\mathcal{F}_{\mathrm{SO}(3)}^{-1} f=\hat{f} \text {. }
$$

The One-Dimensional Radon Transform on SO(3). The initial Radon transform introduced by Funk [3] and Radon [18] was largely generalized by Helgason, see [4, 5] and the references therein. For more specific details concerning the Radon transform on $\mathrm{SO}(3)$ see also [14].

Let $\mathbf{h}, \mathbf{r} \in \mathbb{S}^{2}$. Then the set

$$
G(\mathbf{h}, \mathbf{r})=\{\mathbf{g} \in \mathrm{SO}(3) \mid \mathbf{g h}=\mathbf{r}\}
$$

of all rotations that map the vector $\mathbf{h}$ onto the vector $\mathbf{r}$ defines a geodesic in $\mathrm{SO}(3)$. Moreover, for any geodesic $G \subset \mathrm{SO}(3)$ there is a pair $(\mathbf{h}, \mathbf{r}) \in \mathbb{S}^{2} \times \mathbb{S}^{2}$ such that $G=G(\mathbf{h}, \mathbf{r})$. The pair $(\mathbf{h}, \mathbf{r})$ is well defined up to the symmetry $G(\mathbf{h}, \mathbf{r})=G(-\mathbf{h},-\mathbf{r})$.

Let $\mathbf{h}, \mathbf{r} \in \mathbb{S}^{2}$ and let $\mathbf{g}_{0} \in G(\mathbf{h}, \mathbf{r})$ be an arbitrary rotation mapping $\mathbf{h}$ onto $\mathbf{r}$. Then the geodesic $G(\mathbf{h}, \mathbf{r})$ allows for the parameterization

$$
G(\mathbf{h}, \mathbf{r})=\left\{\operatorname{Rot}_{\mathbf{r}}(\omega) \mathbf{g}_{0} \in \mathrm{SO}(3) \mid \omega \in[0,2 \pi)\right\}
$$

where $\operatorname{Rot}_{\mathbf{r}}(\omega)$ denotes the rotation about the rotational axis $\mathbf{r}$ by the rotation angle $\omega$.

We now step to the definition of the one-dimensional Radon transform on $\mathrm{SO}(3)$ as a bounded operator between $L^{2}(\mathrm{SO}(3))$ and $L^{2}\left(\mathbb{S}^{2} \times \mathbb{S}^{2}\right)$. The strict way to do so is to first define the Radon transform for the class of continuous functions, show that it is bounded with respect to the $L^{2}$-norm and then extend it to a bounded operator between $L^{2}(\mathrm{SO}(3))$ and $L^{2}\left(\mathbb{S}^{2} \times \mathbb{S}^{2}\right)$. However, we only refer to [22] and immediately define the Radon transform in the Hilbert space setting.

Definition 2.3. The (one-dimensional) Radon transform on $\mathrm{SO}(3)$ is defined as the integral operator

$$
\begin{aligned}
\mathcal{R}: L^{2}(\mathrm{SO}(3)) & \rightarrow L^{2}\left(\mathbb{S}^{2} \times \mathbb{S}^{2}\right) \\
(\mathcal{R} f)(\mathbf{h}, \mathbf{r}) & =\frac{1}{2 \pi} \int_{G(\mathbf{h}, \mathbf{r})} f(\mathbf{g}) \mathrm{d} \mathbf{g}, \quad(\mathbf{h}, \mathbf{r}) \in \mathbb{S}^{2} \times \mathbb{S}^{2}
\end{aligned}
$$

For completeness we prove the following Lemma (see also [22]).

Lemma 2.4. Let $l \in \mathbb{N}_{0}$ and $k, k^{\prime}=-l, \ldots, l$. The Radon transform of the Wigner-D function $D_{l}^{k k^{\prime}}$ is given by

$$
\mathcal{R} D_{l}^{k k^{\prime}}(\mathbf{h}, \mathbf{r})=\frac{2 \pi}{l+\frac{1}{2}} \mathcal{Y}_{l}^{k^{\prime}}(\mathbf{h}) \overline{\mathcal{Y}_{l}^{k}(\mathbf{r})}, \quad \mathbf{h}, \mathbf{r} \in \mathbb{S}^{2}
$$


Proof. For arbitrary $l \in \mathbb{N}_{0}, k, k^{\prime}=-l, \ldots, l$ we obtain by equation (2.4)

$$
\begin{aligned}
\mathcal{R} D_{l}^{k k^{\prime}}(\mathbf{h}, \mathbf{r}) & =\frac{1}{2 \pi} \int_{G(\mathbf{h}, \mathbf{r})} D_{l}^{k k^{\prime}}(\mathbf{g}) \mathrm{d} \mathbf{g} \\
& =\frac{1}{2 \pi} \int_{G(\mathbf{h}, \mathbf{r})} \int_{\mathbb{S}^{2}} \mathcal{Y}_{l}^{k^{\prime}}\left(\mathbf{g}^{-1} \boldsymbol{\xi}\right) \overline{\mathcal{Y}_{l}^{k}(\boldsymbol{\xi})} \mathrm{d} \boldsymbol{\xi} \mathrm{dg} \\
& =\frac{1}{2 \pi} \int_{\mathbb{S}^{2}} \mathcal{Y}_{l}^{k^{\prime}}(\boldsymbol{\xi}) \int_{G(\mathbf{h}, \mathbf{r})} \overline{\mathcal{Y}_{l}^{k}(\mathbf{g} \boldsymbol{\xi})} \mathrm{dg} \mathrm{d} \boldsymbol{\xi} .
\end{aligned}
$$

Since we have for any $\boldsymbol{\xi}, \mathbf{h}, \mathbf{r} \in \mathbb{S}^{2}$ and $\mathbf{g}_{0} \in G(\mathbf{h}, \mathbf{r})$,

$$
\left\{\mathbf{g} \boldsymbol{\xi} \in \mathbb{S}^{2} \mid \mathbf{g} \in G(\mathbf{h}, \mathbf{r})\right\}=\left\{\operatorname{Rot}_{\mathbf{r}}(\omega) \mathbf{g}_{0} \boldsymbol{\xi} \in \mathbb{S}^{2} \mid \omega \in[0,2 \pi)\right\}
$$

the inner integral rewrites as

$$
\frac{1}{2 \pi} \int_{G(\mathbf{h}, \mathbf{r})} \overline{\mathcal{Y}_{l}^{k}(\mathbf{g} \boldsymbol{\xi})} \mathrm{dg}=\frac{1}{2 \pi} \int_{0}^{2 \pi} \overline{\mathcal{Y}_{l}^{k}\left(\operatorname{Rot}_{\mathbf{r}}(\omega) \mathbf{g}_{0} \boldsymbol{\xi}\right)} \mathrm{d} \omega=\mathcal{P}_{l}\left(\mathbf{r} \cdot \mathbf{g}_{0} \boldsymbol{\xi}\right) \overline{\mathcal{Y}_{l}^{k}(\mathbf{r})}
$$

Here we have applied the spherical mean value theorem (cf. [2, eq. 3.6.15]). Together with (2.8) and the Funk-Hecke theorem (cf. [2, Th. 3.6.1]) we obtain

$$
\mathcal{R} D_{l}^{k k^{\prime}}(\mathbf{h}, \mathbf{r})=\int_{\mathbb{S}^{2}} \mathcal{Y}_{l}^{k^{\prime}}(\boldsymbol{\xi}) \mathcal{P}_{l}(\mathbf{h} \cdot \boldsymbol{\xi}) \overline{\mathcal{Y}_{l}^{k}(\mathbf{r})} \mathrm{d} \boldsymbol{\xi}=\frac{2 \pi}{l+\frac{1}{2}} \mathcal{Y}_{l}^{k^{\prime}}(\mathbf{h}) \overline{\mathcal{Y}_{l}^{k}(\mathbf{r})}
$$

As a direct consequence of the above lemma we obtain the following characterization of the range of the Radon transform $\mathcal{R}$ on $\mathrm{SO}(3)$.

Lemma 2.5. The range of the Radon transform $\mathcal{R}$ is the subspace of all functions $P \in L^{2}\left(\mathbb{S}^{2} \times\right.$ $\left.\mathbb{S}^{2}\right)$ that have a Fourier expansion of the form

$$
P=\sum_{\left(l, k, k^{\prime}\right) \in J} \hat{P}\left(l, k, k^{\prime}\right) \mathcal{Y}_{l}^{k^{\prime}}\left(\circ_{1}\right) \overline{\mathcal{Y}_{l}^{k}\left(\circ_{2}\right)}
$$

with Fourier coefficients $\hat{P}\left(l, k, k^{\prime}\right),\left(l, k, k^{\prime}\right) \in J$ satisfying the summation property

$$
\sum_{\left(l, k, k^{\prime}\right) \in J}(2 l+1)\left|\hat{P}\left(l, k, k^{\prime}\right)\right|^{2}<\infty
$$

In particular, any function $P \in \mathcal{R} L^{2}(\mathrm{SO}(3))$ in the range of the Radon transform possesses the symmetry property

$$
P(\mathbf{h}, \mathbf{r})=P(-\mathbf{h},-\mathbf{r}), \quad \mathbf{h}, \mathbf{r} \in \mathbb{S}^{2}
$$

For the specific subspace in $L^{2}\left(\mathbb{S}^{2} \times \mathbb{S}^{2}\right)$ spanned by the range of the Radon transform we define the following Fourier transform. 
Definition 2.6. By the operator

$$
\mathcal{F}_{\mathcal{R}}: \ell^{2}(J) \rightarrow L^{2}\left(\mathbb{S}^{2} \times \mathbb{S}^{2}\right), \quad \mathcal{F}_{\mathcal{R}} \hat{P}=\sum_{\left(l, k, k^{\prime}\right) \in J} \hat{P}\left(l, k, k^{\prime}\right) \mathcal{Y}_{l}^{k^{\prime}}\left(\circ_{1}\right) \overline{\mathcal{Y}_{l}^{k}\left(\circ_{2}\right)}
$$

we denote the restriction of the Fourier transform in $L^{2}\left(\mathbb{S}^{2} \times \mathbb{S}^{2}\right)$ to the subspace spanned by functions of the form (2.9).

Next we want to derive an equivalent to the classical Fourier slice theorem for the Radon transform in $\mathbb{R}^{d}$. To this end we define the following two operators. The first operator $\mathcal{V}$ is given as

$$
\mathcal{V}: \ell^{2}(J) \rightarrow \ell^{2}\left(I, L^{2}\left(\mathbb{S}^{2}\right)\right), \quad(\mathcal{V} \hat{P})(l, k)=\sum_{k^{\prime}=-l}^{l} \hat{P}\left(l,-k, k^{\prime}\right) \mathcal{Y}_{l}^{k^{\prime}}
$$

and defines for any sequence $\hat{P} \in \ell^{2}(J)$ a sequence of functions $\mathcal{V} \hat{P}(l, k) \in L^{2}\left(\mathbb{S}^{2}\right),(l, k) \in I$. The norm in $\ell^{2}\left(I, L^{2}\left(\mathbb{S}^{2}\right)\right)$ is given as

$$
\|\mathcal{V} \hat{P}\|_{\ell^{2}\left(I, L^{2}\left(\mathbb{S}^{2}\right)\right)}^{2}=\sum_{(l, k) \in I}\|\mathcal{V} \hat{P}(l, k)\|_{L^{2}\left(\mathbb{S}^{2}\right)}^{2}=\sum_{(l, k) \in I} \int_{0}^{\pi} \int_{0}^{2 \pi}|\mathcal{V} \hat{P}(l, k)(\theta, \rho)|^{2} \mathrm{~d} \rho \sin \theta \mathrm{d} \theta .
$$

The second operator $\mathcal{M}: \ell^{2}(J) \rightarrow \ell^{2}(J)$ we define as the multiplication operator

$$
\mathcal{M} \hat{f}\left(l, k, k^{\prime}\right)=\frac{\pi}{\sqrt{2 l+1}} \hat{f}\left(l, k, k^{\prime}\right), \quad\left(l, k, k^{\prime}\right) \in J .
$$

Theorem 2.7 (Fourier slice). The Radon transform $\mathcal{R}$ on $\mathrm{SO}(3)$ is a multiplication operator in Fourier space

$$
\mathcal{R}=\mathcal{F}_{\mathcal{R}} \mathcal{M} \mathcal{F}_{\mathrm{SO}(3)}^{-1}
$$

Moreover, we have the decomposition

$$
\mathcal{F}_{\mathcal{R}}=\mathcal{F}_{\mathbb{S}^{2}} \mathcal{V}
$$

which finally leads to

$$
\mathcal{R}=\mathcal{F}_{\mathbb{S}^{2}} \mathcal{V} \mathcal{M} \mathcal{F}_{\mathrm{SO}(3)}^{-1}
$$

Proof. The first decomposition (2.13) is a direct consequence of Lemma 2.4. In order to prove the decomposition (2.14) we consider an arbitrary Fourier sequence $\hat{P} \in \ell^{2}(J)$ and verify

$$
\begin{aligned}
\mathcal{F}_{\mathbb{S}^{2}} \mathcal{V} \hat{P} & =\sum_{(l, k) \in I}(\mathcal{V} \hat{P})(l, k) \mathcal{Y}_{l}^{k}=\sum_{\left(l, k, k^{\prime}\right) \in J} \hat{P}\left(l,-k, k^{\prime}\right) \mathcal{Y}_{l}^{k^{\prime}} \mathcal{Y}_{l}^{k} \\
& =\sum_{\left(l, k, k^{\prime}\right) \in J} \hat{P}\left(l, k, k^{\prime}\right) \mathcal{Y}_{l}^{k^{\prime}} \overline{\mathcal{Y}_{l}^{k}}=\mathcal{F}_{\mathcal{R}} \hat{P}
\end{aligned}
$$


The decomposition of the Radon transform $\mathcal{R}$ in Theorem 2.7 can be expressed in a commutative diagram as

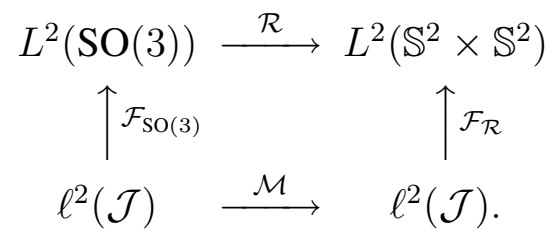

The Inverse Radon Transform. By Lemma 2.4 the inverse Radon transform

$$
\mathcal{R}^{-1}: L^{2}\left(\mathbb{S}^{2} \times \mathbb{S}^{2}\right) \rightarrow L^{2}(\mathrm{SO}(3))
$$

is an unbounded operator and hence its numerical evaluation is an ill posed problem. Regularization of the ill posed problem means to approximate the unbounded operator $\mathcal{R}^{-1}$ by a bounded one. For this purpose we first define two approximations of the inverse Fourier transform $\mathcal{F}_{\mathcal{R}}^{-1}$.

The next result concerns minimization problems in discrete norms in $\ell^{2}$, where $\ell^{2}$ is either $\ell^{2}(I)$ or $\ell^{2}(J)$. Therefore, we define the weighted norm $\|w\|_{\mathcal{W}}, w \in \ell^{2}$ for any nonnegative operator $\mathcal{W}: \ell^{2} \rightarrow \ell^{2}$ called weight as

$$
\|w\|_{\mathcal{W}}=\left\|\mathcal{W}^{1 / 2} w\right\|_{2}
$$

Let $\Gamma \subset \mathbb{S}^{2} \times \mathbb{S}^{2}$ be a finite subset of nodes and let $L \in \mathbb{N}_{0}$. Then we define for any continuous function $P \in \mathcal{C}\left(\mathbb{S}^{2} \times \mathbb{S}^{2}\right)$ the set of Fourier sequences

$$
\begin{aligned}
\Omega_{L, \Gamma}(P)=\left\{\omega \in \ell^{2}(J) \mid\right. & \omega\left(l, k, k^{\prime}\right)=0 \text { for } l>L \text { and } \\
& \left.\left(\mathcal{F}_{\mathcal{R}} \omega\right)(\mathbf{h}, \mathbf{r})=P(\mathbf{h}, \mathbf{r}) \text { for }(\mathbf{h}, \mathbf{r}) \in \Gamma\right\} .
\end{aligned}
$$

Lemma 2.8. Let $\Gamma \subset \mathbb{S}^{2} \times \mathbb{S}^{2}$ be a finite subset of nodes and let $L \in \mathbb{N}_{0}$ such that for any $P \in \mathcal{C}\left(\mathbb{S}^{2} \times \mathbb{S}^{2}\right)$ the set $\Omega_{L, \Gamma}(P)$ is not empty. Then the minimization problem

$$
\tilde{\omega}=\underset{\omega \in \Omega_{L, \Gamma}(P)}{\operatorname{argmin}}\|\omega\|_{\mathcal{W}}^{2}
$$

has a unique solution for any positive operator $\mathcal{W}$ and defines a linear, bounded operator

$$
\mathcal{T}_{L, \Gamma}^{E}: \mathcal{C}\left(\mathbb{S}^{2} \times \mathbb{S}^{2}\right) \rightarrow \ell^{2}(J), \quad \mathcal{T}_{L, \Gamma}^{E} P=\tilde{\omega} .
$$

Proof. Existence and uniqueness of a solution of minimization problem (2.16) follows from the fact that $\|\circ\|_{\mathcal{W}}^{2}$ is a strictly convex functional on the convex set $\Omega_{L, \Gamma}(P)$. Since the set $\Omega_{L, \Gamma}(P)$ is finite dimensional the solution of the minimization problem (2.16) coincides with the solution of the corresponding normal equations of second kind (cf. [20]). In particular, the solution depends linearly on the given continuous function $P$. Since the operator $\mathcal{T}_{L, \Gamma}^{E}$ is finite dimensional it is bounded. 
Replacing the normal equations of second kind by normal equations of first kind the proof of the next lemma is analogous to the previous proof.

Lemma 2.9. Assume that a finite set of nodes $\Gamma \subset \mathbb{S}^{2} \times \mathbb{S}^{2}$ and a polynomial degree $L \in \mathbb{N}_{0}$ are chosen such that for any $P \in \mathcal{C}\left(\mathbb{S}^{2} \times \mathbb{S}^{2}\right)$ the set of Fourier coefficients $\Omega_{L, \Gamma}(P)$ contains at most one element. Then the minimization problem

$$
\tilde{\omega}=\underset{\left\{\omega \in \ell^{2}(J) \mid \omega(l)=0 \text { for } l>L\right\}}{\operatorname{argmin}}\left\|\left(\mathcal{F}_{\mathcal{R}} \omega\right)(\Gamma)-P(\Gamma)\right\|_{2}^{2}+\|\omega\|_{\mathcal{W}}^{2}
$$

has a unique solution for any positive operator $\mathcal{W}$ and defines a linear, bounded operator

$$
\mathcal{T}_{L, \Gamma}^{R}: \mathcal{C}\left(\mathbb{S}^{2} \times \mathbb{S}^{2}\right) \rightarrow \ell^{2}(J), \quad \mathcal{T}_{L, \Gamma}^{R} P=\tilde{\omega}
$$

Obviously a necessary condition for the existence of the first operator is $|\Gamma| \leq\left|J_{L}\right|$ whereas the existence of the operator $\mathcal{T}_{L, \Gamma}^{R}$ requires $|\Gamma| \geq\left|J_{L}\right|$. In the general case of arbitrarily scattered nodes $\Gamma$ one can expect the operators $\mathcal{T}_{L, \Gamma}^{E}$ and $\mathcal{T}_{L, \Gamma}^{R}$ to be well defined if $|\Gamma|<\left|J_{L}\right|$ or $|\Gamma|>\left|J_{L}\right|$, respectively. A more precise result depending on the set of nodes $\Gamma$ will be given in Lemma 3.9.

Definition 2.10. Let $\Gamma \subset \mathbb{S}^{2} \times \mathbb{S}^{2}$ be a finite subset of nodes and let $L \in \mathbb{N}_{0}$ be a polynomial degree. If the operator $\mathcal{T}_{L, \Gamma}^{E}$ exists then we construct the operator

$$
\mathcal{R}_{L, \Gamma}^{E}: \mathcal{C}\left(\mathbb{S}^{2} \times \mathbb{S}^{2}\right) \rightarrow \mathcal{C}(\mathrm{SO}(3)), \quad \mathcal{R}_{L, \Gamma}^{E}=\mathcal{F}_{\mathrm{SO}(3)} \mathcal{M}^{-1} \mathcal{T}_{L, \Gamma}^{E}
$$

and if the operator $\mathcal{T}_{L, \Gamma}^{R}$ exists then we construct the operator

$$
\mathcal{R}_{L, \Gamma}^{R}: \mathcal{C}\left(\mathbb{S}^{2} \times \mathbb{S}^{2}\right) \rightarrow \mathcal{C}(\mathrm{SO}(3)), \quad \mathcal{R}_{L, \Gamma}^{R}=\mathcal{F}_{\mathrm{SO}(3)} \mathcal{M}^{-1} \mathcal{T}_{L, \Gamma}^{R}
$$

The operators $\mathcal{R}_{L, \Gamma}^{E}$ and $\mathcal{R}_{L, \Gamma}^{R}$ are finite dimensional approximations of the inverse Radon transform $\mathcal{R}^{-1}$ which we will use for the numerical inversion. They share the characteristic that for any function $P \in \mathcal{C}\left(\mathbb{S}^{2} \times \mathbb{S}^{2}\right)$ the functions $\mathcal{R}_{L, \Gamma}^{E} P$ and $\mathcal{R}_{L, \Gamma}^{R} P$, respectively, depend only on the values of the function $P$ at the set of nodes $\Gamma \subset \mathbb{S}^{2} \times \mathbb{S}^{2}$. The subscript $L \in \mathbb{N}_{0}$ indicates that the range of both operators contains only functions of polynomial degree $L$. The following theorem gives an estimate on the quality of approximation of the operators $\mathcal{R}_{L, \Gamma}^{E}$ and $\mathcal{R}_{L, \Gamma}^{R}$.

Theorem 2.11. Let $\Gamma \subset \mathbb{S}^{2} \times \mathbb{S}^{2}$ be a finite subset of nodes and let $L \in \mathbb{N}_{0}$ be a polynomial degree such that the operator $\mathcal{R}_{L, \Gamma}^{E}$ is well defined. Then we have for any $P \in \mathcal{C}\left(\mathbb{S}^{2} \times \mathbb{S}^{2}\right)$ the equality

$$
\left\|\mathcal{R}^{-1} P-\mathcal{R}_{L, \Gamma}^{E} P\right\|_{L^{2}(\mathrm{SO}(3))}=\left\|\hat{P}-\mathcal{T}_{L, \Gamma}^{E} P\right\|_{\mathcal{M}^{-2}},
$$

where $\mathcal{M}$ is the multiplication operator defined in (2.12).

If the set of nodes $\Gamma$ and the polynomial degree L are chosen such that the operator $\mathcal{R}_{L, \Gamma}^{R}$ is well defined then an analogous equality is satisfied, i.e.,

$$
\left\|\mathcal{R}^{-1} P-\mathcal{R}_{L, \Gamma}^{R} P\right\|_{L^{2}(\mathrm{SO}(3))}=\left\|\hat{P}-\mathcal{T}_{L, \Gamma}^{R} P\right\|_{\mathcal{M}^{-2}} .
$$


Proof. Since $\mathcal{F}_{\mathrm{SO}(3)}$ is an isomorphism we have by Theorem 2.7

$$
\begin{aligned}
\left\|\mathcal{R}^{-1} P-\mathcal{R}_{L, \Gamma}^{E} P\right\|_{L^{2}(\mathrm{SO}(3))} & =\left\|\mathcal{F}_{\mathrm{SO}(3)} \mathcal{M}^{-1} \mathcal{F}_{\mathcal{R}}^{-1} P-\mathcal{F}_{\mathrm{SO}(3)} \mathcal{M}^{-1} \mathcal{T}_{L, \Gamma}^{E} P\right\|_{L^{2}(\mathrm{SO}(3))} \\
& =\left\|\mathcal{M}^{-1} \mathcal{F}_{\mathcal{R}}^{-1} P-\mathcal{M}^{-1} \mathcal{T}_{L, \Gamma}^{E} P\right\|_{2} \\
& =\left\|\hat{P}-\mathcal{T}_{L, \Gamma}^{E} P\right\|_{\mathcal{M}^{-2}}
\end{aligned}
$$

The second equality follows analogously.

\section{Discrete Operators}

This section is devoted to the discrete theory. Our main goal is to develop a fast numerical algorithm for the evaluation of the operators $\mathcal{R}_{L, \Gamma}^{E}$ and $\mathcal{R}_{L, \Gamma}^{R}$. Therefore we first introduce discrete versions of the Fourier transforms in $L^{2}\left(\mathbb{S}^{2}\right)$ and in $\mathcal{R} L^{2}(\mathrm{SO}(3))$, respectively.

Discrete Fourier Transforms. Let $P \in \bigoplus_{l=0}^{L} \operatorname{Harm}_{l}\left(\mathbb{S}^{2}\right)$ be a spherical polynomial of degree $L \in \mathbb{N}_{0}$. Then we use for the finite Fourier sequence $\hat{P}$ of $P$ the vector notation $\hat{\mathbf{P}} \in \mathbb{C}^{I_{L}}$ with $\hat{\mathbf{P}}_{l, k}=\hat{P}(l, k)$ for $(l, k) \in I_{L}$, where the length of the vector is $\left|I_{L}\right|$. According to [8], we consider the evaluation of the function $P$ at a list of arbitrary nodes given its vector of Fourier coefficients.

Definition 3.1. [Discrete spherical Fourier transform] Let $\boldsymbol{\xi}=\left(\boldsymbol{\xi}_{1}, \ldots, \boldsymbol{\xi}_{N}\right)$ be a vector of $N \in \mathbb{N}$ arbitrary nodes $\boldsymbol{\xi}_{j} \in \mathbb{S}^{2}$ and let $\hat{\mathbf{P}} \in \mathbb{C}^{I_{L}}$ be a vector of Fourier coefficients associated to a spherical polynomial of degree $L \in \mathbb{N}_{0}$. Then the linear operator

$$
\mathbf{F}_{L, \boldsymbol{\xi}}: \mathbb{C}^{I_{L}} \rightarrow \mathbb{C}^{N}, \quad\left[\mathbf{F}_{L, \boldsymbol{\xi}} \hat{\mathbf{P}}\right]_{j}=\sum_{(l, k) \in I_{L}} \hat{\mathbf{P}}_{l, k} \mathcal{Y}_{l}^{k}\left(\boldsymbol{\xi}_{j}\right), \quad j=1, \ldots, N
$$

is called discrete spherical Fourier transform. Its adjoint operator

$$
\mathbf{F}_{L, \boldsymbol{\xi}}^{H}: \mathbb{C}^{N} \rightarrow \mathbb{C}^{I_{L}}, \quad\left[\mathbf{F}_{L, \boldsymbol{\xi}}^{H} \mathbf{c}\right]_{l, k}=\sum_{j=1}^{N} \mathbf{c}_{j} \overline{\mathcal{Y}_{l}^{k}\left(\boldsymbol{\xi}_{j}\right)}, \quad(l, k) \in I_{L},
$$

is called adjoint discrete spherical Fourier transform.

A naive implementation of the discrete spherical Fourier transform and of its adjoint transform for $N \in \mathbb{N}$ arbitrary nodes and for polynomial degree $L \in \mathbb{N}_{0}$ requires $\mathcal{O}\left(N L^{2}\right)$ flops. However, there exist much faster algorithms. The algorithm described in [12] and [7] calculates both transforms in an approximate way with numerical complexity of $\mathcal{O}\left(L^{2} \log ^{2} L+N\right)$ flops. We refer to this algorithm as the nonequispaced fast spherical Fourier transform (NFSFT). An implementation of this algorithm is availably as a part of the NFFT-library [8].

Analogously to the spherical case, we use for the Fourier sequence $\hat{f}$ of a polynomial $f \in$ $L^{2}(\mathrm{SO}(3))$ of degree $L \in \mathbb{N}_{0}$ the vector notation $\hat{\mathbf{f}} \in \mathbb{C}^{J_{L}}, \hat{\mathbf{f}}_{l, k, k^{\prime}}=\hat{f}\left(l, k, k^{\prime}\right),\left(l, k, k^{\prime}\right) \in J_{L}$. 
Definition 3.2. [Discrete Fourier transform on $\mathrm{SO}(3)$ ] Let $\mathbf{g}=\left(\mathrm{g}_{1}, \ldots, \mathbf{g}_{N}\right)$ be a vector of $N \in \mathbb{N}$ arbitrary nodes $\mathbf{g}_{i} \in \mathrm{SO}(3)$ and let $\hat{\mathbf{f}} \in \mathbb{C}^{J_{L}}$ be the vector of Fourier coefficients associated to a polynomial $f \in L^{2}(\mathrm{SO}(3))$ of degree $L \in \mathbb{N}_{0}$. Then the linear operator

$$
\mathbf{F}_{L, \mathbf{g}}: \mathbb{C}^{J_{L}} \rightarrow \mathbb{C}^{N}, \quad\left[\mathbf{F}_{L, \mathbf{g}} \hat{\mathbf{f}}\right]_{j}=\sum_{\left(l, k, k^{\prime}\right) \in J_{L}} \frac{\left(l+\frac{1}{2}\right)^{\frac{1}{2}}}{2 \pi} \hat{\mathbf{f}}_{l, k, k^{\prime}} D_{l}^{k k^{\prime}}\left(\mathbf{g}_{j}\right), \quad j=1, \ldots, N
$$

is called discrete Fourier transform on $\mathrm{SO}(3)$. Its adjoint operator

$$
\mathbf{F}_{L, \mathbf{g}}^{H}: \mathbb{C}^{N} \rightarrow \mathbb{C}^{J_{L}}, \quad\left[\mathbf{F}_{L, \mathbf{g}}^{H} \mathbf{c}\right]_{l, k, k^{\prime}}=\frac{\left(l+\frac{1}{2}\right)^{\frac{1}{2}}}{2 \pi} \sum_{j=1}^{N} \mathbf{c}_{j} \overline{D_{l}^{k k^{\prime}}\left(\mathbf{g}_{j}\right)},
$$

$\left(l, k, k^{\prime}\right) \in J_{L}$, is called adjoint discrete Fourier transform on $\mathrm{SO}(3)$.

We notice that a naive implementation of the discrete Fourier transform on $\mathrm{SO}(3)$ and of its adjoint transform for $N \in \mathbb{N}$ arbitrary nodes and for a polynomial degree $L \in \mathbb{N}_{0}$ has the numerical complexity of $\mathcal{O}\left(N L^{3}\right)$ flops. An $\mathcal{O}\left(L^{4}\right)$ algorithm for the case of regular aligned nodes with $N=\mathcal{O}\left(L^{3}\right)$ was proposed in [11], based on a separation of variables. This algorithm was generalized in [17] to an $\mathcal{O}\left(L^{3} \log ^{2} L+N\right)$ algorithm that works for arbitrary nodes, based on the nonequispaced fast Fourier transform (cf. [16]).

For our purposes we introduce a discrete version $\mathbf{F}_{L, \Gamma}$ of the operator $\mathcal{F}_{\mathcal{R}}$ as defined in Definition 2.6 with respect to a finite set of nodes $\Gamma \subset \mathbb{S}^{2} \times \mathbb{S}^{2}$. Therefore, we abbreviate the Fourier sequence $\hat{P}$ of a polynomial $P \in \mathcal{R} L^{2}(\mathrm{SO}(3))$ of degree $L \in \mathbb{N}_{0}$ by the vector $\hat{\mathbf{P}} \in \mathbb{C}^{J_{L}}, \hat{\mathbf{P}}_{l, k, k^{\prime}}=\hat{P}\left(l, k, k^{\prime}\right),\left(l, k, k^{\prime}\right) \in J_{L}$.

Definition 3.3. Let $L \in \mathbb{N}_{0}$ be a certain polynomial degree and let $\Gamma \subset \mathbb{S}^{2} \times \mathbb{S}^{2}$ be a finite set of nodes of the form

$$
\begin{aligned}
\Gamma= & \left(\left(\mathbf{h}_{1}, \mathbf{r}_{1,1}\right),\left(\mathbf{h}_{1}, \mathbf{r}_{1,2}\right), \ldots,\left(\mathbf{h}_{1}, \mathbf{r}_{1, N_{1}}\right),\right. \\
& \left.\left(\mathbf{h}_{2}, \mathbf{r}_{2,1}\right),\left(\mathbf{h}_{2}, \mathbf{r}_{2,2}\right), \ldots,\left(\mathbf{h}_{2}, \mathbf{r}_{2, N_{2}}\right), \ldots,\left(\mathbf{h}_{N}, \mathbf{r}_{N, N_{N}}\right)\right),
\end{aligned}
$$

where $N, N_{i} \in \mathbb{N}, i=1, \ldots, N$. Then we define the operator $\mathbf{F}_{L, \Gamma}$ as

$$
\mathbf{F}_{L, \Gamma}: \mathbb{C}^{J_{L}} \rightarrow \mathbb{C}^{\Gamma}, \quad\left[\mathbf{F}_{L, \Gamma} \mathbf{w}\right]_{i j}=\sum_{\left(l, k, k^{\prime}\right) \in J_{L}} \mathbf{w}_{l, k, k^{\prime}} \mathcal{Y}_{l}^{k^{\prime}}\left(\mathbf{h}_{i}\right) \overline{\mathcal{Y}_{l}^{k}\left(\mathbf{r}_{i j}\right)},
$$

with $i=1, \ldots, N, j=1, \ldots, N_{i}$, and call

$$
\mathbf{F}_{L, \Gamma}^{H}: \mathbb{C}^{\Gamma} \rightarrow \mathbb{C}^{J_{L}}, \quad\left[\mathbf{F}_{L, \Gamma}^{H} \mathbf{u}\right]_{l, k, k^{\prime}}=\sum_{i=1}^{N} \sum_{j=1}^{N_{i}} \mathbf{u}_{i, j} \overline{\mathcal{Y}_{l}^{k^{\prime}}\left(\mathbf{h}_{i}\right)} \mathcal{Y}_{l}^{k}\left(\mathbf{r}_{i j}\right),
$$

with $\left(l, k, k^{\prime}\right) \in J_{L}$, its adjoint operator. 
Discrete Fourier Slice Theorem. Now we are going to give a discrete analogue to the Fourier slice Theorem 2.7 by decomposing the operator $\mathbf{F}_{L, \Gamma}$ into $N$ independent discrete spherical Fourier transforms and an operator that is almost diagonal and acts separately on the harmonic spaces.

The sum in (3.1) can be rewritten in the following way:

$$
\left[\mathbf{F}_{L, \Gamma} \mathbf{w}\right]_{i, j}=\sum_{l=0}^{L} \sum_{k=-l}^{l} \mathbf{v}_{i, l, k} \mathcal{Y}_{l}^{k}\left(\mathbf{r}_{i j}\right)=\left[\mathbf{F}_{L, \mathbf{r}_{i}} \mathbf{v}_{i}\right]_{j}, \quad i=1, \ldots, N, j=1, \ldots, N_{i}
$$

with

$$
\mathbf{v}_{i, l, k}=\sum_{k^{\prime}=-l}^{l} \mathbf{w}_{l,-k, k^{\prime}} \mathcal{Y}_{l}^{k^{\prime}}\left(\mathbf{h}_{i}\right), \quad i=1, \ldots N,(l, k) \in I_{L} .
$$

Here we have used the vector notation $\mathbf{v}_{i}=\left(\mathbf{v}_{i, l, k}\right)_{(l, k) \in I_{L}} \in \mathbb{C}^{I_{L}}, i=1, \ldots, N$. The decomposition of the operator $\mathbf{F}_{L, \Gamma}$ will be based on the following matrices. We define the permutation matrix $\Pi \in \mathbb{C}^{N\left|I_{L}\right| \times N\left|I_{L}\right|}$ by

$$
[\mathbf{\Pi} \mathbf{x}]_{i,(l, k)}=\mathbf{x}_{(l,-k), i}
$$

where $\mathbf{x} \in \mathbb{C}^{\left|I_{L}\right| N}$. Furthermore, we define the matrices $\mathbf{V}_{l} \in \mathbb{C}^{N \times(2 l+1)}, l=0, \ldots, N$, and $\mathbf{V} \in \mathbb{C}^{\left|I_{L}\right| N \times\left|J_{L}\right|}$ as

$$
\mathbf{V}_{l}=\left(\mathcal{Y}_{l}^{k}\left(\mathbf{h}_{i}\right)\right)_{i=1, \ldots, N, k=-l, \ldots, l}, \quad \mathbf{V}=\left(\left[\mathbf{V}_{l}\right]_{i, \tilde{k}^{\prime}} \delta_{l, \tilde{l}^{\prime}} \delta_{k, \tilde{k}}\right)_{\left((l, k) \in I_{L}, i=1, \ldots, N\right),\left(\left(\tilde{l}, \tilde{k}, \tilde{k}^{\prime}\right) \in J_{L}\right)} .
$$

The matrices $\mathbf{V}_{l}, l=0, \ldots, L$, and $\mathbf{V}$ may also be written as

$$
\mathbf{V}_{l}=\left(\begin{array}{ccc}
\mathcal{Y}_{l}^{-l}\left(\mathbf{h}_{1}\right) & \ldots & \mathcal{Y}_{l}^{l}\left(\mathbf{h}_{1}\right) \\
\vdots & \ddots & \vdots \\
\mathcal{Y}_{l}^{-l}\left(\mathbf{h}_{N}\right) & \ldots & \mathcal{Y}_{l}^{l}\left(\mathbf{h}_{N}\right)
\end{array}\right), \quad \mathbf{V}=\left(\begin{array}{ccc}
\mathbf{I}_{1} \otimes \mathbf{V}_{0} & & \\
& \ddots & \\
& & \mathbf{I}_{2 L+1} \otimes \mathbf{V}_{L}
\end{array}\right)
$$

where the matrices $\mathbf{I}_{2 l+1}, l \in \mathbb{N}_{0}$ are the identity matrices of $\mathbb{C}^{(2 l+1) \times(2 l+1)}$ and $\otimes$ denotes the Kronecker product.

Finally, we assume the set of nodes $\Gamma \subset \mathbb{S}^{2} \times \mathbb{S}^{2}$ to be indexed as specified in Definition 3.3 and additionally introduce the notation $\mathbf{r}_{i}=\left(\mathbf{r}_{i, 1}, \ldots, \mathbf{r}_{i, N_{i}}\right)$.

Theorem 3.4. The operator $\mathbf{F}_{L, \Gamma}$ satisfies the decomposition

$$
\mathbf{F}_{L, \Gamma}=\left(\begin{array}{ccc}
\mathbf{F}_{L, \mathbf{r}_{1}} & & \\
& \ddots & \\
& & \mathbf{F}_{L, \mathbf{r}_{N}}
\end{array}\right) \Pi \mathbf{I V}
$$

Proof. Let $L \in \mathbb{N}_{0}$ and let $\Gamma \subset \mathbb{S}^{2} \times \mathbb{S}^{2}$ be a set of nodes as specified in Definition 3.3. Then we have, for any vector of Fourier coefficients $\mathbf{w} \in \mathbb{C}^{J_{L}}$, the equality

$$
[\mathbf{\Pi V \mathbf { w }}]_{i,(l, k)}=[\mathbf{V w}]_{(l,-k), i}=\sum_{k^{\prime}=-l}^{l} \mathbf{w}_{l,-k, k^{\prime}} \mathcal{Y}_{l}^{k^{\prime}}\left(\mathbf{h}_{i}\right)=\mathbf{v}_{i, l, k}, \quad i=1, \ldots, N,(l, k) \in I_{L} .
$$




\section{Discrete Operators}

Thus we obtain for any $j=1, \ldots, N_{i}$,

$$
\begin{aligned}
{\left[\mathbf{F}_{L, \mathbf{r}_{i}} \mathbf{v}_{i}\right]_{j} } & =\sum_{(l, k) \in I_{L}}\left[\mathbf{v}_{i}\right]_{l, k} \mathcal{Y}_{l}^{k}\left(\mathbf{r}_{i j}\right) \\
& =\sum_{\left(l, k, k^{\prime}\right) \in J_{L}} \mathbf{w}_{l,-k, k^{\prime}} \mathcal{Y}_{l}^{k^{\prime}}\left(\mathbf{h}_{i}\right) \mathcal{Y}_{l}^{k}\left(\mathbf{r}_{i j}\right) \\
& =\sum_{\left(l, k, k^{\prime}\right) \in J_{L}} \mathbf{w}_{l, k, k^{\prime}} \mathcal{Y}_{l}^{k^{\prime}}\left(\mathbf{h}_{i}\right) \overline{\mathcal{Y}_{l}^{k}\left(\mathbf{r}_{i j}\right)}=\left[\mathbf{F}_{L, \Gamma} \mathbf{w}\right]_{i j} .
\end{aligned}
$$

The decomposition of the operator $\mathbf{F}_{L, \Gamma}$ leads to a fast algorithm for its computation. Let $\mathbf{w} \in \mathbb{C}^{J_{L}}$ and $\left(\rho_{i}, \theta_{i}\right) \in[0,2 \pi) \times[0, \pi]$ be the polar coordinates of the node $\mathbf{h}_{i} \in \mathbb{S}^{2}, i=$ $1, \ldots, N$. Then the vectors $\mathbf{v}_{i} \in \mathbb{C}^{I_{L}}, i=1, \ldots, N$, given in (3.4) have, for $(l, k) \in I_{L}$, the form

$$
\begin{aligned}
{\left[\mathbf{v}_{i}\right]_{l, k}=} & \sqrt{\frac{2 l+1}{4 \pi}} \sum_{k^{\prime}=-l}^{l} \mathbf{w}_{l,-k, k^{\prime}} \mathcal{P}_{l}^{\left|k^{\prime}\right|}\left(\cos \theta_{i}\right) \mathrm{e}^{\mathrm{i} k^{\prime} \rho_{i}} \\
= & \sqrt{\frac{2 l+1}{4 \pi}} \mathbf{w}_{l,-k, 0} \mathcal{P}_{l}^{0}\left(\cos \theta_{i}\right) \\
& +\sqrt{\frac{2 l+1}{4 \pi}} \sum_{k^{\prime}=1}^{l}\left(\mathbf{w}_{l,-k, k^{\prime}} \mathrm{e}^{\mathrm{i} k^{\prime} \rho_{i}}+\mathbf{w}_{l,-k,-k^{\prime}} \mathrm{e}^{-\mathrm{i} k^{\prime} \rho_{i}}\right) \mathcal{P}_{l}^{k^{\prime}}\left(\cos \theta_{i}\right) .
\end{aligned}
$$

Utilizing the three term recurrence relation (2.1) of the associated Legendre polynomials, Algorithm 1 presents an implementation for the calculation of the vectors $\mathbf{v}_{i} \in \mathbb{C}^{I_{L}}$ given in (3.4) with numerical complexity of $\mathcal{O}\left(L^{3}\right)$ flops. In order to reduce memory consumption Algorithm 1 does not precompute all values $P_{l}^{k^{\prime}}\left(\cos \left(\theta_{i}\right)\right)$ but organizes the recurrence scheme such that only four values of $P_{l}^{k^{\prime}}$ have to kept in memory. Together with Theorem 3.4 we conclude that utilizing the NFSFT for the calculation of $\mathbf{F}_{L, \mathbf{r}_{i}} \mathbf{v}_{i}, i=1, \ldots, N$, i.e. the sum (3.3), we obtain the overall complexity of $\mathcal{O}\left(N L^{3}+|\Gamma|\right)$ flops for the computation of $\mathbf{F}_{L, \Gamma} \mathbf{w}$.

Let $\mathbf{u}=\left(\mathbf{u}_{1}^{T}, \ldots, \mathbf{u}_{N}^{T}\right)^{T}, \mathbf{u}_{i} \in \mathbb{C}^{N_{i}}, i=1, \ldots, N$. For the adjoint operator $\mathbf{F}_{L, \Gamma}^{H}$ we rewrite (3.2) in the following way

$$
\left[\mathbf{F}_{L, \Gamma}^{H} \mathbf{u}\right]_{l, k, k^{\prime}}=\sum_{i=1}^{N} \tilde{\mathbf{v}}_{i, l,-k} \overline{\mathcal{Y}_{l}^{k^{\prime}}\left(\mathbf{h}_{i}\right)}, \quad\left(l, k, k^{\prime}\right) \in J_{L},
$$

with

$$
\tilde{\mathbf{v}}_{i, l, k}=\sum_{j=1}^{N_{i}} \mathbf{u}_{i, j} \overline{\mathcal{Y}_{l}^{k}\left(\mathbf{r}_{i, j}\right)}=\mathbf{F}_{L, \mathbf{r}_{i}}^{H} \mathbf{u}_{i}, \quad i=1, \ldots, N,(l, k) \in I_{L} .
$$

Again we use the vector notation $\tilde{\mathbf{v}}_{i}=\left(\tilde{\mathbf{v}}_{i, l, k}\right)_{(l, k) \in I_{L}} \in \mathbb{C}^{I_{L}}, i=1, \ldots, N$, and mention that, given the vectors $\mathbf{u}_{i} \in \mathbb{C}^{N_{i}}$, the vectors $\tilde{\mathbf{v}}_{i}, i=1, \ldots, N$, can be computed by $N$ adjoint NFSFTs with the numerical complexity of $\mathcal{O}\left(N L^{2} \log ^{2} L+|\Gamma|\right)$ flops. 


\section{Discrete Operators}

It remains to compute (3.6). Therefore, we define the vectors $\tilde{\mathbf{w}}^{i} \in \mathbb{C}^{J_{L}}, i=1, \ldots, N$, as

$$
\tilde{\mathbf{w}}_{l, k, k^{\prime}}^{i}=\tilde{\mathbf{v}}_{i, l,-k} \overline{\mathcal{Y}_{l}^{k^{\prime}}\left(h_{i}\right)}=\sqrt{\frac{2 l+1}{4 \pi}}\left[\tilde{\mathbf{v}}_{i}\right]_{l,-k} \mathrm{e}^{-i k^{\prime} \rho_{i}} \mathcal{P}_{l}^{\left|k^{\prime}\right|}\left(\cos \theta_{i}\right), \quad\left(l, k, k^{\prime}\right) \in J_{L}
$$

the computation of which is implemented in Algorithm 2. Performing this algorithm $N$ times and summing up the vectors $\tilde{\mathbf{w}}^{i} \in \mathbb{C}^{J_{L}}, i=1, \ldots, N$, results in the numerical complexity of $\mathcal{O}\left(N L^{3}\right)$ flops. Hence, we have the overall numerical complexity of $\mathcal{O}\left(N L^{3}+|\Gamma|\right)$ flops for the application of the adjoint operator $\mathbf{F}_{L, \Gamma}^{H}$.

Summarizing the above we obtain the following result.

Theorem 3.5. Let $L \in \mathbb{N}_{0}$ be a polynomial degree, and let $\Gamma \subset \mathbb{S}^{2} \times \mathbb{S}^{2}$ be a set of nodes as specified in Definition 3.3. Then, for any vector $\mathbf{w} \in \mathbb{C}^{J_{L}}$ and any vector $\mathbf{u} \in \mathbb{C}^{\Gamma}$, the approximative computation of $\mathbf{F}_{L, \Gamma} \mathbf{W}$ and $\mathbf{F}_{L, \Gamma}^{H} \mathbf{u}$ using the NFSFT and the Algorithms 1 and 2 , respectively, has the complexity of $\mathcal{O}\left(N L^{3}+|\Gamma|\right)$ flops, whereas a naive algorithm has the complexity of $\mathcal{O}\left(|\Gamma| L^{3}\right)$ flops.

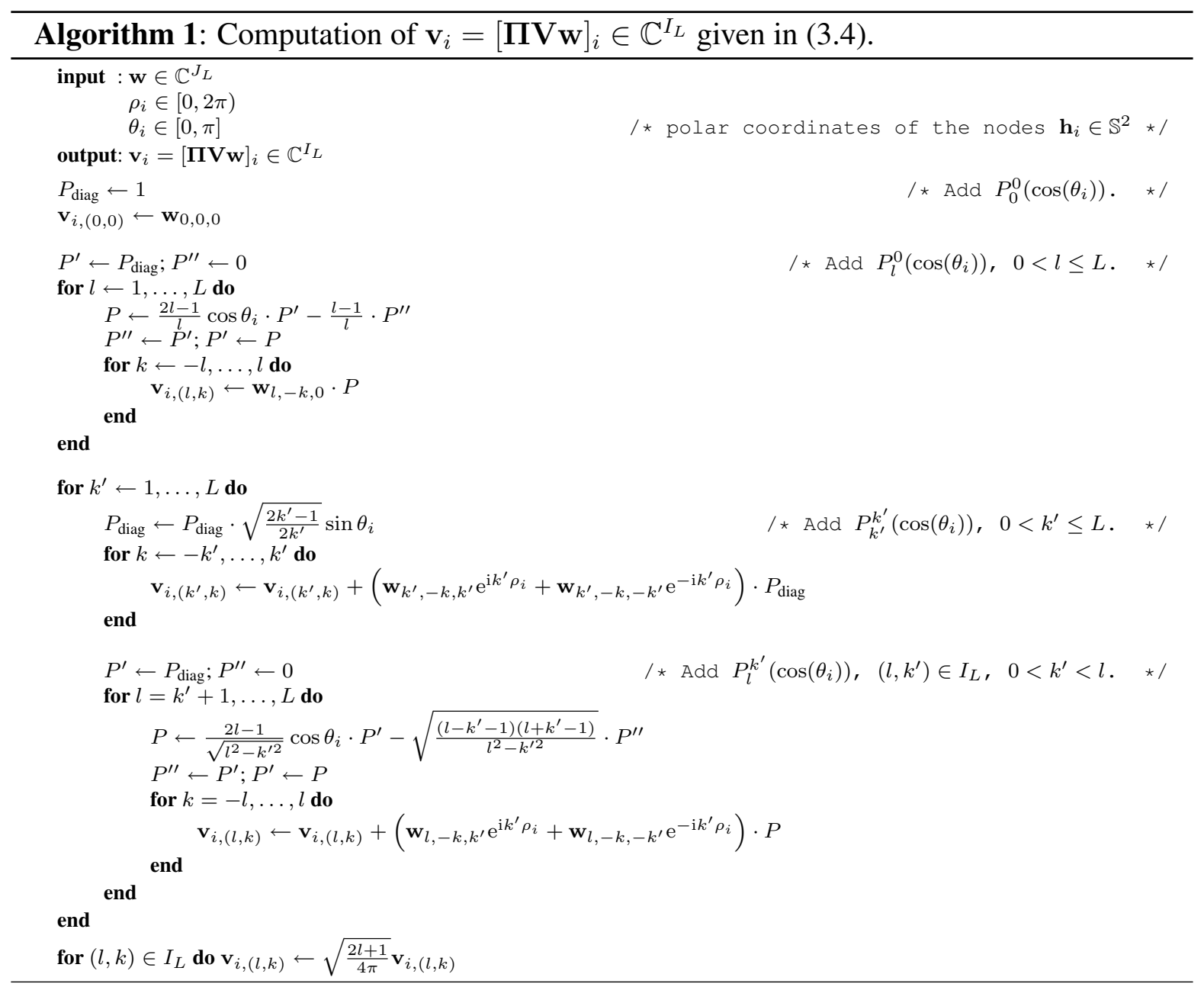




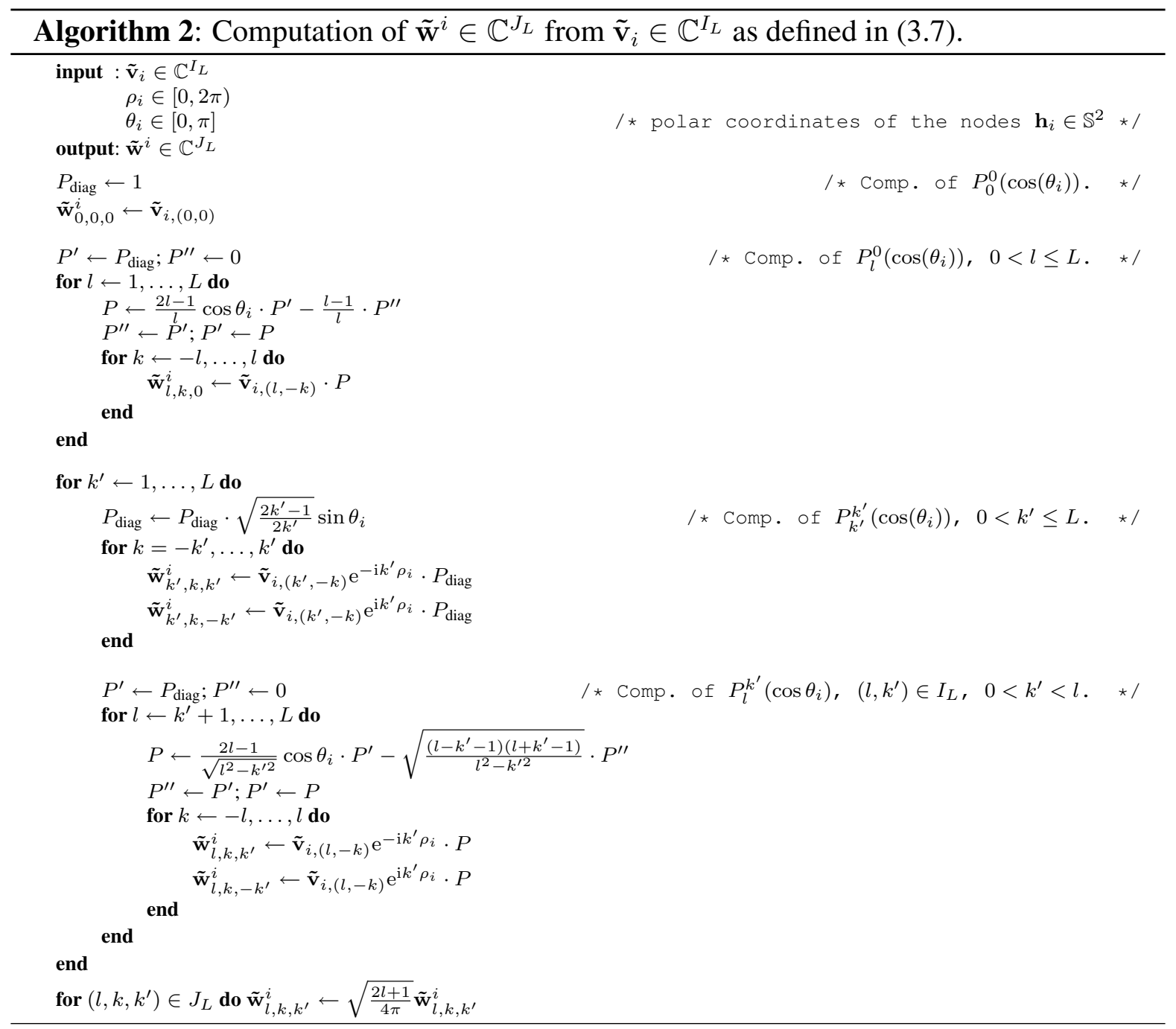

Numerical Inversion of the Radon Transform. Next we are going to combine the discrete Fourier transforms introduced in the previous section in order to derive numerical methods for the inversion of the Radon transform on $\mathrm{SO}(3)$. By Theorem 2.11 the operators

$$
\mathcal{R}_{L, \Gamma}^{E}=\mathcal{F}_{\mathrm{SO}(3)} \mathcal{M}^{-1} \mathcal{T}_{L, \Gamma}^{E} \quad \text { and } \quad \mathcal{R}_{L, \Gamma}^{R}=\mathcal{F}_{\mathrm{SO}(3)} \mathcal{M}^{-1} \mathcal{T}_{L, \Gamma}^{R}
$$

provide finite dimensional approximations of the inverse Radon transform $\mathcal{R}^{-1}$. Since a fast algorithm for the Fourier transform $\mathcal{F}_{\mathrm{SO}(3)}$ exists (cf. [11, 17]), we focus on the computation of the operators $\mathcal{T}_{L, \Gamma}^{E}$ and $\mathcal{T}_{L, \Gamma}^{R}$. To be precise we emphasize now the dependence of the operators $\mathcal{T}_{L, \Gamma}^{E}$ and $\mathcal{T}_{L, \Gamma}^{R}$ on the weights $\mathcal{W}: \ell^{2}(J) \rightarrow \ell^{2}(J)$ and introduce $\mathbf{W} \in \mathbb{C}^{J_{L} \times J_{L}}$ as its canonical restriction to an operator acting between $\mathbb{C}^{J_{L}}$ and $\mathbb{C}^{J_{L}}$.

Restricting the minimization problems (2.16) and (2.18) to their effective domains and ranges Lemma 2.8 and Lemma 2.9 may be rewritten using the discrete operators $\mathbf{F}_{L, \Gamma}$ and W. 
Lemma 3.6. Let $\Gamma \subset \mathbb{S}^{2} \times \mathbb{S}^{2}$ be a finite set of nodes and let $L \in \mathbb{N}_{0}$ be a polynomial degree such that the operator $\mathcal{T}_{L, \Gamma}^{E}$ is well defined. Then for any function $P \in C\left(\mathbb{S}^{2} \times \mathbb{S}^{2}\right)$ the minimization problem

$$
\mathbf{w}^{E}=\underset{\left\{\mathbf{w} \in \mathbb{C}^{J} L \mid \mathbf{F}_{L, \Gamma} \mathbf{w}=P(\Gamma)\right\}}{\operatorname{argmin}}\|\mathbf{w}\|_{\mathbf{W}}^{2}
$$

has a unique solution $\mathbf{w}^{E} \in \mathbb{C}^{J_{L}}$, which coincides with $\mathcal{T}_{L, \Gamma}^{E} P$ in the sense of

$$
\mathbf{w}_{l, k, k^{\prime}}^{E}=\mathcal{T}_{L, \Gamma}^{E} P\left(l, k, k^{\prime}\right), \quad\left(l, k, k^{\prime}\right) \in J_{L} .
$$

Lemma 3.7. Assume that the set of nodes $\Gamma \subset \mathbb{S}^{2} \times \mathbb{S}^{2}$ and the polynomial degree $L \in \mathbb{N}_{0}$ are chosen such that the operator $\mathcal{T}_{L, \Gamma}^{R}$ is well defined. Then for any function $P \in C\left(\mathbb{S}^{2} \times \mathbb{S}^{2}\right)$ the minimization problem

$$
\mathbf{w}^{R}=\underset{\mathbf{w} \in \mathbb{C}^{J_{L}}}{\operatorname{argmin}}\left\|\mathbf{F}_{L, \Gamma} \mathbf{w}-P(\Gamma)\right\|_{2}^{2}+\|\mathbf{w}\|_{\mathbf{w}}^{2}
$$

has a unique solution $\mathbf{w}^{R} \in \mathbb{C}^{J_{L}}$, which coincides with $\mathcal{T}_{L, \Gamma}^{R} P$ in the sense of (3.9).

The minimization problems (3.8) and (3.10) can be numerically solved by the CGNE and the CGNR algorithm, respectively, cf. e.g. [20], which results in a fast numerical method for the approximate calculation of the inverse Fourier transform $\mathcal{F}_{\mathcal{R}}$ in the range of the Radon transform. More precisely, we obtain from Theorem 3.5 the following result.

Theorem 3.8. Let $L \in \mathbb{N}_{0}$ be a polynomial degree and assume that the set of nodes $\Gamma \in \mathbb{S}^{2} \times \mathbb{S}^{2}$ has the structure as described in Definition 3.3. Then $\mathbf{w}^{R}=\mathcal{T}_{L, \Gamma}^{R} P$ or $\mathbf{w}^{E}=\mathcal{T}_{L, \Gamma}^{E} P$ can be computed by the CGNR or CGNE algorithm, respectively. Moreover, both algorithms can be implemented such that each iteration step has the numerical complexity of $\mathcal{O}\left(N L^{3}+|\Gamma|\right)$ flops.

The complexity of $\mathcal{O}\left(N L^{3}+|\Gamma|\right)$ flops for a single iteration step using the NFSFT compares to the complexity of $\mathcal{O}\left(L^{3}|\Gamma|\right)$ flops of a naive implementation. Hence, the asymptotic superiority of our algorithm depends on the quotient $\mathcal{O}(|\Gamma| / N)$. Next we are going to show that this quotient can not be better then $\mathcal{O}\left(L^{2}\right)$.

Theorem 3.9. For the matrix $\mathbf{F}_{L, \Gamma}$ to be injective it is necessary that $|\Gamma|=\sum_{i=1}^{N} N_{i} \geq\left|J_{L}\right|$ and that $N \geq 2 L+1$.

Proof. The first condition follows immediately from the dimension of the matrix $\mathbf{F}_{L, \Gamma}$. For the second condition we use the decomposition (3.5) and observe that the matrix $\mathbf{V} \in \mathbb{C}^{N\left|I_{L}\right| \times\left|J_{L}\right|}$ has full row rank $\left|J_{L}\right|$ if and only if each block $\mathbf{V}_{l}$ has full row rank $2 l+1$. In particular, we obtain for the last block $\mathbf{V}_{L}$ the condition $N \geq 2 L+1$.

If $\mathcal{O}(|\Gamma|)=\mathcal{O}\left(\left|J_{L}\right|\right)$ and $\mathcal{O}(N)=\mathcal{O}(L)$, our NFFT based algorithm has the numerical complexity of $\mathcal{O}\left(|\Gamma|^{4 / 3}\right)$ flops per iteration which compares to the numerical complexity of $\mathcal{O}\left(|\Gamma|^{2}\right)$ flops of a naive implementation.

Once the minimization problem (3.8) or (3.10) has been solved the numerical evaluation of $\mathcal{R}^{-1} P(\mathbf{g}) \approx \mathbf{F}_{L, \mathbf{g}}^{H} \mathbf{M} \mathbf{w}^{E / R}$ for a vector of rotations $\mathbf{g}=\left(\mathbf{g}_{1}, \ldots, \mathbf{g}_{K}\right)$ has the complexity of $\mathcal{O}\left(L^{3} \log ^{2} L+K\right)$ flops by applying a fast Fourier transform on $\operatorname{SO}(3)$ [17]. Here the operator $\mathbf{M} \in \mathbb{C}^{J_{L} \times J_{L}}$ denotes the canonical restriction of the multiplication operator $\mathcal{M}: \ell^{2}(J) \rightarrow$ $\ell^{2}(J)$. 


\section{Numerical Tests}

Let $f \in \bigoplus_{l=0}^{L} \operatorname{Harm}_{l}(\mathrm{SO}(3))$ be an arbitrary function with polynomial degree at most $L \in \mathbb{N}_{0}$, let $\Gamma \subset \mathbb{S}^{2} \times \mathbb{S}^{2}$ be a finite set of nodes and let $\mathbf{P}=\mathcal{R} f(\Gamma) \in \mathbb{C}^{|\Gamma|}$ be a sampling of the Radon transform of the function $f$.

Let furthermore $\mathbf{w} \in \mathbb{C}^{J_{L}}$ be an approximation of the Fourier coefficients of $\mathcal{R} f$ computed by one of the algorithms introduced in this paper. Then the approximation error for the original function $f$ is

$$
\left\|f-\mathcal{F}_{\mathrm{SO}(3)} \mathbf{M w}\right\|_{L^{2}(\mathrm{SO}(3))}=\|\hat{\mathbf{f}}-\mathbf{M w}\|_{2},
$$

where

$$
\mathbf{M}=\operatorname{diag}(\mathbf{m}), \quad \mathbf{m} \in \mathbb{R}^{J_{L}}, \mathbf{m}_{l, k, k^{\prime}}=\frac{\pi}{\sqrt{2 l+1}},\left(l, k, k^{\prime}\right) \in J_{L} .
$$

is the discrete version of the multiplication operator $\mathcal{M}$ introduced in 2.7. In this section we analyze the error (4.1) in dependency of the set of nodes $\Gamma_{N, N^{\prime}}$ and the polynomial degree $L$ in numerical experiments.

General Setting. First of all we construct approximative equidistributions $\mathbf{h}_{i} \in \mathbb{S}^{2}, i=$ $1, \ldots, 2 N$ and $\mathbf{r}_{j} \in \mathbb{S}^{2}, j=1, \ldots, N^{\prime}, N^{\prime} \approx(N / 2)^{2}$ on the sphere following [2] such that $\mathbf{h}_{i}=-\mathbf{h}_{i+N}, i=1, \ldots, N$, and define the set of nodes $\Gamma_{N, N^{\prime}} \subset \mathbb{S}^{2} \times \mathbb{S}^{2}$ as

$$
\Gamma_{N, N^{\prime}}=\left\{\left(\mathbf{h}_{i}, \mathbf{r}_{j}\right) \in \mathbb{S}^{2} \times \mathbb{S}^{2} \mid i=1, \ldots, N, j=1, \ldots, N^{\prime}\right\} .
$$

The reason to include only one of the vectors $\mathbf{h}_{i},-\mathbf{h}_{i}, i=1, \ldots, N$, at time into the set of nodes $\Gamma_{N, N^{\prime}}$ is the symmetry property (2.10) of the function we are going to sample.

Second, we randomly generate Fourier coefficients $\hat{f}\left(l, k, k^{\prime}\right) \in \mathbb{C},\left(l, k, k^{\prime}\right) \in J_{L}$ by taking a $\left|J_{L}\right|$-dimensional random sample of the uniform distribution on the disc $\mathbb{D}=\{z \in \mathbb{C} \mid$ $|z| \leq 1\}$ and dividing it by $(l+1)^{2}, l=0, \ldots, L$. This ensures a decay of the Fourier coefficients of $\mathcal{O}\left(l^{-2}\right)$. In general this decay rate assured continuity of the corresponding function and its Radon transform (cf. [6, Lemma 2.22]).

Third, we evaluate the Radon transform $\mathcal{R} f$ of the function $f$ specified by its Fourier coefficients $\hat{f}$ at the nodes $\Gamma_{N, N^{\prime}}$, i.e., we calculate

$$
\mathbf{P}_{i j}=P\left(\mathbf{h}_{i}, \mathbf{r}_{j}\right)=\sum_{\left(l, k, k^{\prime}\right) \in J_{L}} \frac{\pi}{\sqrt{2 l+1}} \hat{f}\left(l, k, k^{\prime}\right) \mathcal{Y}_{l}^{k^{\prime}}\left(\mathbf{h}_{i}\right) \overline{\mathcal{Y}_{l}^{k}\left(\mathbf{r}_{j}\right)} .
$$

In a fourth step we apply the CGNE and CGNR algorithms to the minimization problems (3.8) and (3.10), respectively, and calculate the vectors $\mathbf{w}^{E}=\mathcal{T}_{L, \Gamma_{N, N^{\prime}}}^{E} P$ and $\mathbf{w}^{R}=\mathcal{T}_{L, \Gamma_{N, N^{\prime}}}^{R} P$. Hereby, we utilize Theorem 3.4 for fast multiplications with the matrix $\mathbf{F}_{L, \Gamma_{N, N^{\prime}}}$. The weight matrix $\mathbf{W} \in \mathbb{C}^{\left|J_{L}\right| \times\left|J_{L}\right|}$ is the zero matrix in the case of the CGNR, and the identity matrix in the case of the CGNE algorithm.

Finally, we calculate the relative residual norms $\left\|\mathbf{P}-\mathbf{F}_{L, \Gamma_{N, N^{\prime}}} \mathbf{w}^{E / R}\right\|_{2} /\|\mathbf{P}\|_{2}$ and the relative $\ell^{2}$-errors in the Fourier space $\left\|\hat{\mathbf{f}}-\mathbf{M w}^{E / R}\right\|_{2} /\|\hat{\mathbf{f}}\|_{2}$ which, according to (4.1), are equal to the relative approximation error $\left\|f-\mathcal{F}_{\mathrm{SO}(3)} \mathcal{M} \mathbf{w}^{E / R}\right\|_{L^{2}(\mathrm{SO}(3))} /\|f\|_{L^{2}(\mathrm{SO}(3))}$ in $L^{2}(\mathrm{SO}(3))$. 
For each choice of $L, N$ and $N^{\prime}$, we repeat the steps above for 10 times and take the geometric means of the resulting relative residual norms and approximation errors, which we call mean relative residual norm and mean relative approximation error, respectively.

Numerical Results. All calculations have been executed on computers of "TSP IT Systeme", each of which has an AMD Athlon 64, 3000+ processor, 512 Mb RAM with SUSE Linux 10.

For the concrete numerical experiment we have chosen the polynomial degrees $L$ between 5 and 80 , and sets of nodes $\Gamma_{N, N^{\prime}}$ with $N=11, \ldots, 308, N^{\prime}=34, \ldots, 23898$. In Figures 4.1 the (mean) relative residual norms and approximation errors for the CGNR based algorithm are plotted for any combination of the polynomial degree $L$ and the set of nodes $\Gamma_{N, N^{\prime}}$.
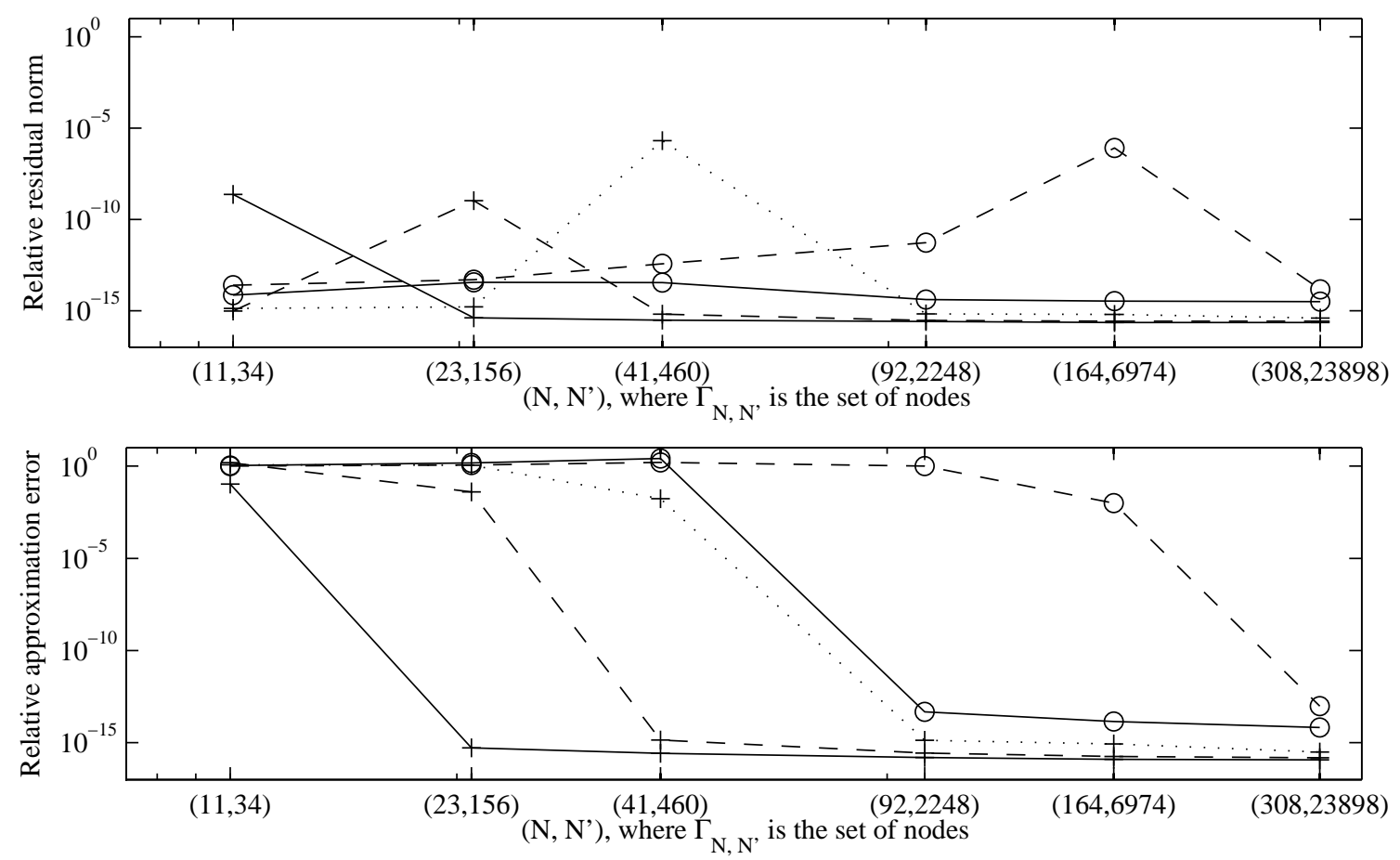

Figure 4.1: The relative residual norm $\left\|\mathbf{P}-\mathbf{F}_{L, \Gamma} \mathbf{w}^{R}\right\|_{2} /\|\mathbf{P}\|_{2}$ in $L^{2}(\operatorname{SO}(3))$ and the relative approximation error $\left\|f-\mathcal{F}_{\mathrm{SO}(3)} \mathcal{M} \mathbf{w}^{R}\right\|_{L^{2}(\mathrm{SO}(3))} /\|f\|_{L^{2}(\mathrm{SO}(3))}$ in $L^{2}(\mathrm{SO}(3))$ of the CGNR based algorithms for polynomial degrees $L=5(\quad), L=10,(\quad) L=20$ ( ), $L=40(\quad), L=80(\quad)$. The dimensions $\left|J_{L}\right| \in\{286,1771,12341,91881,708561\}$ compares to the cardinalities $\left|\Gamma_{N, N^{\prime}}\right| \in\{374,3588,18860,206816,1143736,7360584\}$ of the sets o nodes.

We observe that the relative residual norms in most cases become small showing the convergence of the algorithm. The peaks in the cases of $\left|\Gamma_{N, N^{\prime}}\right| \approx\left|J_{L}\right|$ indicate that the underlying matrices are ill-conditioned. By construction of the sets of nodes the condition $\left|\Gamma_{N, N^{\prime}}\right| \approx\left|J_{L}\right|$ implies in particular $N^{\prime} \approx\left|I_{L}\right|$. Furthermore, we see that the original function $f$ can be reconstructed, provided the number of nodes is large enough. More precisely, we observed that, if $f$ can be reconstructed with a certain set of nodes $\Gamma_{N, N^{\prime}}$, then $f$ can also be reconstructed with 
each set of nodes $\Gamma_{\tilde{N}, \tilde{N}^{\prime}}$ under consideration such that $\tilde{N}>N$ and $\tilde{N}^{\prime}>N^{\prime}$. Interestingly, the CGNR algorithm behaves well even in the cases with $\left|\Gamma_{N, N^{\prime}}\right|<<\left|J_{L}\right|$.

The results for the CGNE based algorithm are not given here. They show up a similar behavior except that the relative residual norms and the approximation error becomes worse if the number of nodes becomes too large. This is due to the fact that the CGNE algorithm requires $\left|\Gamma_{N, N^{\prime}}\right|<\left|J_{L}\right|$.

Runtime. In order to compare the performance of the NFSFT based algorithm with a direct approach we measure the computation time of a forward transform $\mathbf{F}_{L, \Gamma}$ using the NFSFT and a direct implementation, respectively. Using the same parameters $L, N, N^{\prime}$ as in the previous experiment we observe that the NFSFT based algorithm is substantially faster. As Table 4.1 shows the difference in runtime between the NFSFT based algorithm and the direct algorithm increases as the polynomial degree increases.

\begin{tabular}{rrrrr}
\hline \multicolumn{3}{c}{ Parameter } & \multicolumn{2}{c}{ Runtime in sec. } \\
L & N & $N^{\prime}$ & fast alg. & direct alg. \\
\hline 10 & 23 & 156 & 0.25 & 0.61 \\
20 & 41 & 460 & 1.22 & 6.30 \\
40 & 92 & 2248 & 8.38 & 233 \\
80 & 164 & 6974 & 60.5 & 5092 \\
\hline
\end{tabular}

Table 4.1: Runtime comparison between the fast algorithm using the NFSFT and the direct algorithm.

\section{An Application to Texture Analysis}

The Radon transform on $\mathrm{SO}(3)$ is of central importance for the analysis of crystallographic preferred orientations, the technical term of which is texture analysis cf. [24]. It establishes the relationship between the so called orientation density function (ODF) $f: \operatorname{SO}(3) / \mathcal{G} \rightarrow \mathbb{R}$, which models the distribution of crystal orientations within a polycrystalline specimen and the so called pole density function (PDF) $P: \mathbb{S}^{2} / \mathcal{G} \times \mathbb{S}^{2} \rightarrow \mathbb{R}$, which models the distribution of crystallographic lattice planes within the specimen. Here $\mathcal{G} \subset \mathrm{SO}(3)$ denotes a finite subgroup of $\mathrm{SO}(3)$ which represents the crystal symmetries. In terms of the Radon transform the relationship between the ODF $f \in L^{2}(\mathrm{SO}(3) / \mathcal{G})$ and the PDF $P \in L^{2}\left(\mathbb{S}^{2} / \mathcal{G} \times \mathbb{S}^{2}\right)$ of a specimen reads as

$$
P(\mathbf{h}, \mathbf{r})=\frac{1}{2}(\mathcal{R} f(\mathbf{h}, \mathbf{r})+\mathcal{R} f(-\mathbf{h}, \mathbf{r})) .
$$

PDFs can experimentally be sampled by diffraction techniques like X-ray, neutron, or synchrotron diffraction, whereas ODFs cannot directly be measured by these techniques. A central problem in texture analysis is the estimation of the ODF of a specimen given its measured 
PDF cf. [1]. Of particular importance are the lower order Fourier coefficients of the ODF since they characterize the macroscopic properties of the specimen, e.g. the second order Fourier coefficients characterize thermal expansion, optical refraction index, and electrical conductivity whereas the fourth order Fourier coefficients characterize the elastic properties of the specimen cf. [1, sec. 13].

By Proposition 2.4 we have the following relationship between the Fourier coefficients of an ODF $f \in L^{2}(\mathrm{SO}(3) / \mathcal{G})$ and the corresponding PDF $P \in L^{2}\left(\mathbb{S}^{2} / \mathcal{G} \times \mathbb{S}^{2}\right)$,

$$
P=\mathcal{F}_{\mathcal{R}} \mathcal{M}_{\text {even }} \mathcal{F}_{\text {SO }(3)}^{-1} f, \quad \mathcal{M}_{\text {even }} \hat{f}\left(l, k, k^{\prime}\right)= \begin{cases}\frac{\pi}{\sqrt{2 l+1}} \hat{f}\left(l, k, k^{\prime}\right) & l \text { even }, \\ 0 & l \text { odd } .\end{cases}
$$

In particular, the odd order Fourier coefficients of the ODF $f$ cannot be reconstructed from the PDF $P$ without additional modeling assumptions.

In order to apply our algorithm to the PDF - to - ODF inversion problem we fix the trigonal crystal symmetry group $\mathcal{G} \subset \mathrm{SO}(3)$ (cf. [1]) and define a set of $N=37$ different directions

$\mathbf{h}_{i} \in \mathcal{G}\left\{\left(\begin{array}{l}1 \\ 0 \\ 0\end{array}\right),\left(\begin{array}{l}0 \\ 1 \\ 1\end{array}\right),\left(\begin{array}{l}1 \\ 0 \\ 1\end{array}\right),\left(\begin{array}{l}1 \\ 1 \\ 0\end{array}\right),\left(\begin{array}{l}1 \\ 0 \\ 2\end{array}\right),\left(\begin{array}{l}1 \\ 1 \\ 1\end{array}\right),\left(\begin{array}{l}0 \\ 2 \\ 1\end{array}\right),\left(\begin{array}{l}1 \\ 1 \\ 2\end{array}\right),\left(\begin{array}{l}0 \\ 1 \\ 2\end{array}\right),\left(\begin{array}{l}2 \\ 0 \\ 1\end{array}\right),\left(\begin{array}{l}0 \\ 0 \\ 1\end{array}\right)\right\}, \quad i=1, \ldots, N$.

The nodes $\mathbf{r}_{j}, j=1, \ldots, N^{\prime}, N^{\prime}=9791$ we choose independently as an approximative equidistribution on the hemisphere $\mathbb{S}_{+}^{2}$ and define the set of nodes $\Gamma$ analogously as in Section 4 as

$$
\Gamma=\left\{\left(\mathbf{h}_{i}, \mathbf{r}_{j}\right) \mid i=1, \ldots, N, j=1, \ldots, N^{\prime}\right\} .
$$

The construction of the sampling set reflects practical experimental settings that are restricted to many directions $\mathbf{r}_{j}$ but only few directions $\mathbf{h}_{i}$. As a consequence, only the Fourier coefficients of the lowest order can be determined (cf. Theorem 3.9).

Proceeding as in Section 4 we randomly generate Fourier coefficients $\hat{f}\left(l, k, k^{\prime}\right),\left(l, k, k^{\prime}\right) \in$ $J_{L_{0}}$, up to the polynomial degree $L_{0}=128$ with decay rate $\mathcal{O}\left(l^{-4}\right)$ and simulate a sampling of the corresponding PDF by calculating $\mathbf{P}=\mathbf{F}_{L_{0}, \Gamma} \mathcal{M}_{\text {even }} \hat{f}$. For arbitrary non polynomial functions on $\mathrm{SO}(3)$ the decay rate $\mathcal{O}\left(l^{-4}\right)$ of the Fourier coefficients ensures that the function is continuously differentiable. Applying the CGNR algorithm with $L=0,2, \ldots, 32$ to the sample $\mathbf{P} \in \mathbb{R}^{\Gamma}$ we obtain Fourier coefficients $\hat{\mathbf{P}} \in \mathbb{C}^{J_{L}}$. In fact, we use a modified version of the CGNR algorithm which ensures that the components $\hat{\mathbf{P}}_{l, k, k^{\prime}}$ of $\hat{\mathbf{P}}$ with $l$ odd become zero. The weight matrix $\mathbf{W}$ is the zero matrix. By setting

$$
\varepsilon(L, l)=\left(\sum_{k, k^{\prime}=-l}^{l}\left|\hat{f}\left(l, k, k^{\prime}\right)-\frac{\sqrt{2 l+1}}{\pi} \hat{\mathbf{P}}_{l, k, k^{\prime}}^{s}\right|^{2}\right)^{1 / 2}\left(\sum_{k, k^{\prime}=-l}^{l}\left|\hat{f}\left(l, k, k^{\prime}\right)\right|^{2}\right)^{-1 / 2}
$$

we compute the relative error between the calculated and the initial Fourier coefficients of the ODF separately for each harmonic space $\operatorname{Harm}_{l}(\mathrm{SO}(3)), l=0, \ldots, L$. Obviously, we have $\varepsilon(L, l)=1$ in the case that $l>L$ or $l$ is odd. In Figure 5.1a the relative errors $\varepsilon(L, l)$, $l=0,2, \ldots, L$ are plotted for different maximum polynomial degrees $L \in \mathbb{N}$ of calculation.

According to Lemma 3.9 and [1, sec. 4.2.2.1] the maximum polynomial degrees up to which the Fourier coefficients can be reconstructed given the sampling $\mathbf{P}$ is $l=18$. However, our 


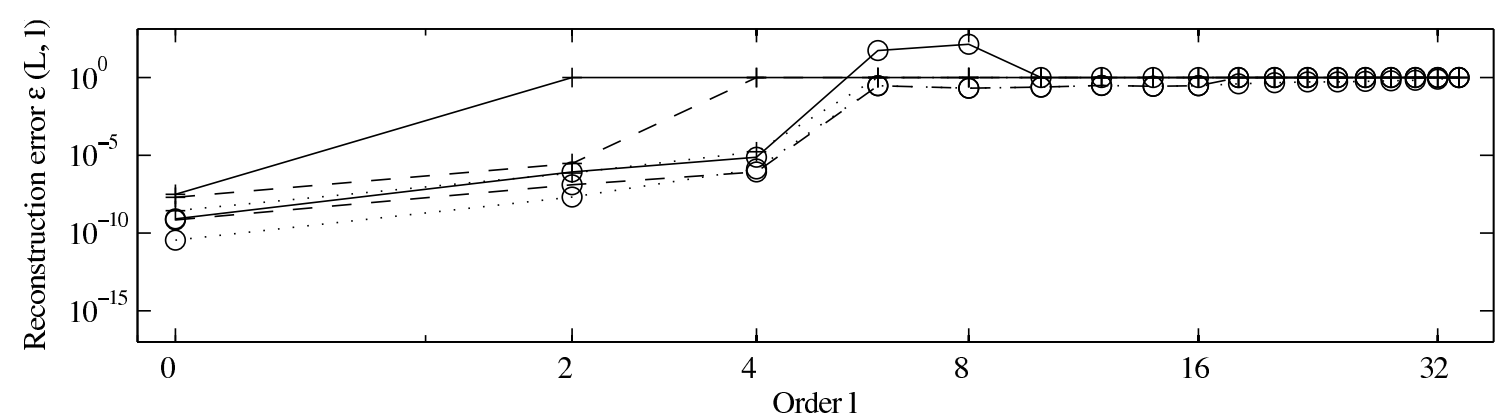

(a) set of nodes $\Gamma$ with $N=37, N^{\prime}=9791$

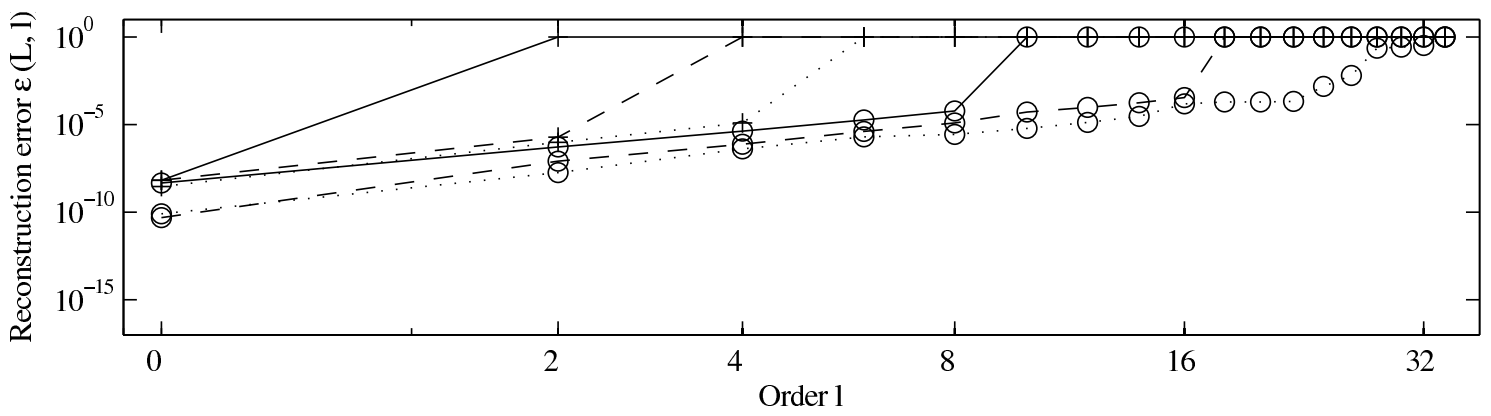

(b) set of nodes $\Gamma$ with $N=73, N^{\prime}=9791$

Figure 5.1: The approximation error $\varepsilon(L, l)$ of the Fourier coefficients of a function with polynomial degree $L_{0}=128$ sampled at a set of nodes $\Gamma$ in dependency of their order $l$ and the total bandwidth used for approximation $L=0(\quad), L=2(\quad), L=4(\quad)$, $L=8(\quad), L=16(\quad), L=32(\quad)$.

numerical experiments show that the polynomial degree up to which the Fourier coefficients are reconstructed is in general lower. In our example the approximation error $\varepsilon(L, l)$ is significantly lower than 1 only for the orders $l=0,2,4$. Interestingly, the approximation error $\varepsilon(L, l)$ decreases as the bandwidth $L=0,2, \ldots, 32$ used for the approximation of the Radon transform increases.

In a second experiment we have chosen cubic crystal symmetry and $N=73$ different directions $\mathbf{h}_{i} \in \mathbb{S}^{2}, i=1, \ldots, N$. For this setting the maximum polynomial degree is 36 . According to Figure $5.1 \mathrm{~b}$ we came quit close to this bound. The observation that $\varepsilon(L, l)$ increases with increasing $l=0,2, \ldots, L$ is due to the decay rate $\mathcal{O}\left(l^{-4}\right)$ of the generated Fourier coefficients $\hat{f}\left(l, k, k^{\prime}\right)$ implying that the denominator in (5.2) decreases as $l$ increases. Again we mention that the approximation error $\varepsilon(L, l), l=0,2, \ldots, 16$ of the low order Fourier coefficients decreases as the bandwidth $L$ increases.

\section{Conclusions}

Analogously to the classical Radon transform in Euclidean spaces the representation of the Radon transform on $\mathrm{SO}(3)$ in Fourier space has been shown to lead to a fast algorithm for its inversion. Primarily the Fourier space representation allows to define a discrete inverse Radon 
transform as the solution of a minimization problem. Applying fast Fourier transforms on $\mathbb{S}^{2}$ and $\mathrm{SO}(3)$ it can iteratively be solved with standard algorithms. Numerical tests have confirmed the convergence of CGNE and CGNR based algorithms for appropriate assumptions.

As with respect to the practical application in texture anaylsis we have shown that the classical "harmonic method" can largely be improved. The first low order Fourier coefficients which are required to compute antipodally symmetric macroscopic specimen properties from the corresponding single crystal properties are better approximated if the polynomial degree of the ansatz function is much larger than commonly used by practitioners of texture analysis. The frequently heard objection that large polynomial degrees result in numerical instability and eventually in unreliable numbers is obsolete.

\section{Acknowledgments}

The authors gratefully acknowledge financial support by Deutsche Forschungsgemeinschaft, grant "high resolution texture analysis" (PR 331/11, SCHA 465/15) and thank the referees and the editor for their helpful comments and valuable suggestions.

\section{References}

[1] H. J. Bunge. Texture Analysis in Material Science. Butterworths, 1982.

[2] W. Freeden, T. Gervens, and M. Schreiner. Constructive Approximation on the Sphere. Oxford University Press, Oxford, 1998.

[3] P. Funk. Über eine geometrische Anwendung der Abelschen Integralgleichung. Mathematische Annalen, 77:129 - 135, 1915.

[4] S. Helgason. Groups and Geometric Analysis. Academic Press, 1984.

[5] S. Helgason. The Radon Transform. Birkhäuser, 2nd edition, 1999.

[6] R. Hielscher. The Radon Transform on the Rotation Group - Inversion and Application to Texture Analysis. Disseratation, Department of Geosciences, University of Technology Freiberg, $2007 . \quad$ "https://fridolin.tufreiberg.de/archiv/html/MathematikHielscherRalf361401.html”.

[7] J. Keiner and D. Potts. Fast evaluation of quadrature formulae on the sphere. Math. Comput., 77:397 - 419, 2008.

[8] J. Keiner, S. Kunis, and D. Potts. NFFT 3.0, C subroutine library. http://www.tu-chemnitz.de/ potts/nfft, 2006.

[9] J. Keiner, S. Kunis, and D. Potts. Efficient reconstruction of functions on the sphere from scattered data. J. Fourier Anal. Appl., 13:435 - 458, 2007. 
[10] U. F. Kocks, C. N. Tomé, H. R. Wenk, and H. Mecking. Texture and Anisotropy. Cambridge University Press, 1998.

[11] P. J. Kostelec and D. N. Rockmore. FFTs on the rotation group. Santa Fe Institute Working Papers Series Paper 03-11-060, 2003.

[12] S. Kunis and D. Potts. Fast spherical Fourier algorithms. J. Comput. Appl. Math., 161: $75-98,2003$.

[13] S. Matthies, G. Vinel, and K. Helmig. Standard Distributions in Texture Analysis, volume 1. Akademie-Verlag Berlin, 1987.

[14] L. Meister and H. Schaeben. A concise quaternion geometry of rotations. MMAS, 28: 101-126, 2004.

[15] F. Natterer and F. Wübbeling. Mathematical Methods in Image Reconstruction. SIAM, Philadelphia, 2000.

[16] D. Potts, G. Steidl, and M. Tasche. Fast Fourier transforms for nonequispaced data: A tutorial. In J. J. Benedetto and P. J. S. G. Ferreira, editors, Modern Sampling Theory: Mathematics and Applications, pages 247 - 270. Birkhäuser, Boston, 2001.

[17] D. Potts, J. Prestin, and A. Vollrath. A fast Fourier algorithm on the rotation group. Preprint A-07-06, Univ. zu Lübeck, 2007.

[18] J. Radon. Über die Bestimmung von Funktionen durch ihre Integralwerte längs gewisser Mannigfaltigkeiten. Ber. Verh. Sächs. Akad. Wiss. Leipzig. Math. Nat. Kl., 69:262 - 277, 1917.

[19] V. Randle and O. Engler. Introduction to Texture Analysis Macrotexture: Microtexture and Orientation Mapping. Gordon and Breach Science Publishers, 2000.

[20] Y. Saad. Iterative Methods for Sparse Linear Systems. PWS Publ., Boston, 1st edition, 1996.

[21] H. Schaeben and K. G. v.d. Boogaart. Spherical harmonics in texture analysis. Tectonophysics, 370:253-268, 2003.

[22] K. G. v.d. Boogaart, R. Hielscher, J. Prestin, and H. Schaeben. Kernel-based methods for inversion of the radon transform on $S O(3)$ and their applications to texture analysis. J. Comput. Appl. Math., 199:122 - 140, 2007.

[23] N. J. Vilenkin and A. U. Klimyk. Representation of Lie Groups and Special Functions, volume 1. Kluwer Academic Publishers, 1991.

[24] H. R. Wenk. Preferred orientation in deformed metals and rocks: An introduction to modern texture analysis. Academic Press, Orlando, 1985. 